Canadian Art Review

\title{
Inversion comique ou critique satirique ? La vue du Capitole de Hieronymus Cock (1562)
}

\section{Denis Ribouillault}

Volume 38, numéro 1, 2013

URI : https://id.erudit.org/iderudit/1066666ar

DOI : https://doi.org/10.7202/1066666ar

Aller au sommaire du numéro

\section{Éditeur(s)}

UAAC-AAUC (University Art Association of Canada | Association d'art des universités du Canada)

ISSN

0315-9906 (imprimé)

1918-4778 (numérique)

Découvrir la revue

Citer cet article

Ribouillault, D. (2013). Inversion comique ou critique satirique ? La vue du Capitole de Hieronymus Cock (1562). RACAR : Revue d'art canadienne / Canadian Art Review, 38(1), 79-96. https://doi.org/10.7202/1066666ar

\section{Résumé de l'article}

Hieronymus Cock's view of the Capitoline Hill, published in his 1562 series on Roman ruins, has long been considered a useful document by historians of art and architecture for the key historical and topographical information it contains on one of Rome's most celebrated sites during the Renaissance. Beyond its documentary nature, which, as will appear, was essentially rhetorical, the view also offers much information as to how a mid-sixteenth-century Flemish artist might perceive Rome's illustrious topography and celebrated ancient statuary. In other words, Cock's engraving enables us to put into practice what may be called an "archaeology of the gaze.” Through previously unnoticed details, Cock invents a comical-verging on the satirical-vision of the antique sculptures proudly displayed on the famous piazza. Such an ironical reversal of Italian classical dignity is typical of the attitude of some contemporary Flemish artists, such as Pieter Bruegel the Elder, who was then close to Cock, and exposes the ambivalent position of some Northern European artists towards the classical tradition and Italian art theory. Finally, the analysis of other engravings of ruins by Hieronymus Cock where two emblematic characters-the draftsman and the kakker (the one who defecates) - appear side by side, sheds light on the origin and possible significance of these comical and subversive details.
Tous droits réservés (C) UAAC-AAUC (University Art Association of Canada | Association d'art des universités du Canada), 2013
Ce document est protégé par la loi sur le droit d'auteur. L’utilisation des services d'Érudit (y compris la reproduction) est assujettie à sa politique d'utilisation que vous pouvez consulter en ligne.

https://apropos.erudit.org/fr/usagers/politique-dutilisation/ 


\title{
Inversion comique ou critique satirique? La vue du Capitole de Hieronymus Cock (1562)
}

\author{
Denis Ribouillault, Université de Montréal
}

\begin{abstract}
Hieronymus Cock's view of the Capitoline Hill, published in his 1562 series on Roman ruins, has long been considered a useful document by historians of art and architecture for the key historical and topographical information it contains on one of Rome's most celebrated sites during the Renaissance. Beyond its documentary nature, which, as will appear, was essentially rhetorical, the view also offers much information as to how a mid-sixteenth-century Flemish artist might perceive Rome's illustrious topography and celebrated ancient statuary. In other words, Cock's engraving enables us to put into practice what may be called an "archaeology of the gaze."Through previously unnoticed details, Cock invents a comical — verging on the satirical—vision of the antique sculptures proudly displayed on the famous piazza. Such an ironical reversal of Italian classical dignity is typical of the attitude of some contemporary Flemish artists, such as Pieter Bruegel the Elder, who was then close to Cock, and exposes the ambivalent position of some Northern European artists towards the classical tradition and Italian art theory. Finally, the analysis of other engravings of ruins by Hieronymus Cock where two emblematic characters - the draftsman and the kakker (the one who defecates) - appear side by side, sheds light on the origin and possible significance of these comical and subversive details.
\end{abstract}

L La vue du Capitole gravée par Hieronymus Cock et publiée en 1562 est une image largement diffusée par les historiens de l'architecture et de la topographie de Rome à la Renaissance (fig. 1). Figurant parmi les plus anciennes représentations qui nous soient parvenues de la célèbre place, elle documente un état particulièrement intéressant de son histoire, soit la construction de la double rampe d'accès au palais des Sénateurs, attribuée à Michel-Ange. Dans les innombrables publications qui mentionnent la vue, l'unique et obsédante préoccupation des historiens est celle de la datation : 1547 et 1548-49 reviennent le plus souvent ; c'est la période durant laquelle fut construit le grand escalier achevé en 15541 .

L'utilisation que l'on a faite de la gravure de Cock pour dater l'état d'un site historique célèbre est symptomatique, me semble-t-il, de la manière dont sont, encore aujourd'hui, considérées les vues topographiques ou vedute dans l'histoire de l'architecture, attitude que résume avec autorité James Ackerman dans un compte-rendu de 1954 :

The chronological problems of the Capitoline are typical of the Renaissance in that they can be solved only with the aid of graphic documents : views, maps, plans. Yet the scientific use of this kind of document has not yet become standard procedure ; in this case errors could have been avoided if vedute had been accorded as much respect as archival material. Examples of the more important undated Capitoline views may serve to illustrate the advantages of precise analysis $^{2}$.

La qualité de " document " attribuée aux vues topographiques de la Renaissance est cependant hautement problématique étant donné leur nature fortement artificielle et composite. Timothy Riggs, dans son étude magistrale sur Hieronymus Cock, insiste sur le fait que rien n'indique que les vues de ruines et monuments romains publiées par Cock furent réalisées « d'après nature » et que les dessins, pour la plupart perdus, sur lesquels ils sont basés furent certainement retravaillés dans l'atelier ${ }^{3}$. Même son séjour à Rome, généralement daté entre 1546 et 1548 , n'est pas clairement documenté et sa datation repose en partie sur celle de la vue du Capitole et la supposition extrêmement fragile qu'elle fut élaborée par l'artiste lui-mêmét. Déjà à la fin du XIX siècle, Adolf Michaelis doutait de la " véracité " de la vue : "Il me semble que la vue ne montre pas un moment précis de la reconstruction, mais qu'il s'agit d'une vue décidément idéale " 5 . Cette opinion est encore plus évidente si l'on considère la vue de Cock au sein de la série à laquelle elle appartient, soit la troisième et dernière série des ruines romaines publiée par Cock en 1562, les deux premières étant parues respectivement en 1551 et $1561^{6}$. Christian Hülsen notait, il y longtemps déjà, et avec une déception non déguisée, que « les vues de Cock [...], à part leur état avancé de décrépitude (Verschüttung), ne nous apprennent pratiquement rien "sur la topographie de Rome ${ }^{7}$. Considérer comme un " document " fiable, au même titre qu'un document d'archives, ce type de vue topographique représente donc un paradoxe intéressant. Il s'explique par la ténacité du préjugé consistant à associer les vues flamandes à l'idée de " réalisme topographique ", préjugé forgé, en réalité, par les auteurs mêmes de ces images. En effet, les titres des séries de ruines romaines de Cock insistent tous sur l'idée que les vues furent réalisées «d'après nature " et que leur principal but était "d'imiter la réalité " (ad veri imitationem). Cette insistance était en fait toute rhétorique et fonctionnait comme un argument de vente auprès des artistes flamands restés au pays - les principaux destinataires de ces séries de ruines et monuments romains - qui disposaient ainsi d'un répertoire varié de motifs classicisants pour leurs tableaux et leurs compositions. Le titre de la série de 1562, dans laquelle Cock se présente comme " peintre " et implicitement inventeur des compositions (Hieronymus Cock piktor Anverpianus excudebat), suggère que les vues furent réalisées de manière " aussi vraie que splendide" (non minus vere quam pulcherrime deformatas) ${ }^{8}$. Afin de renforcer cette dimension de 


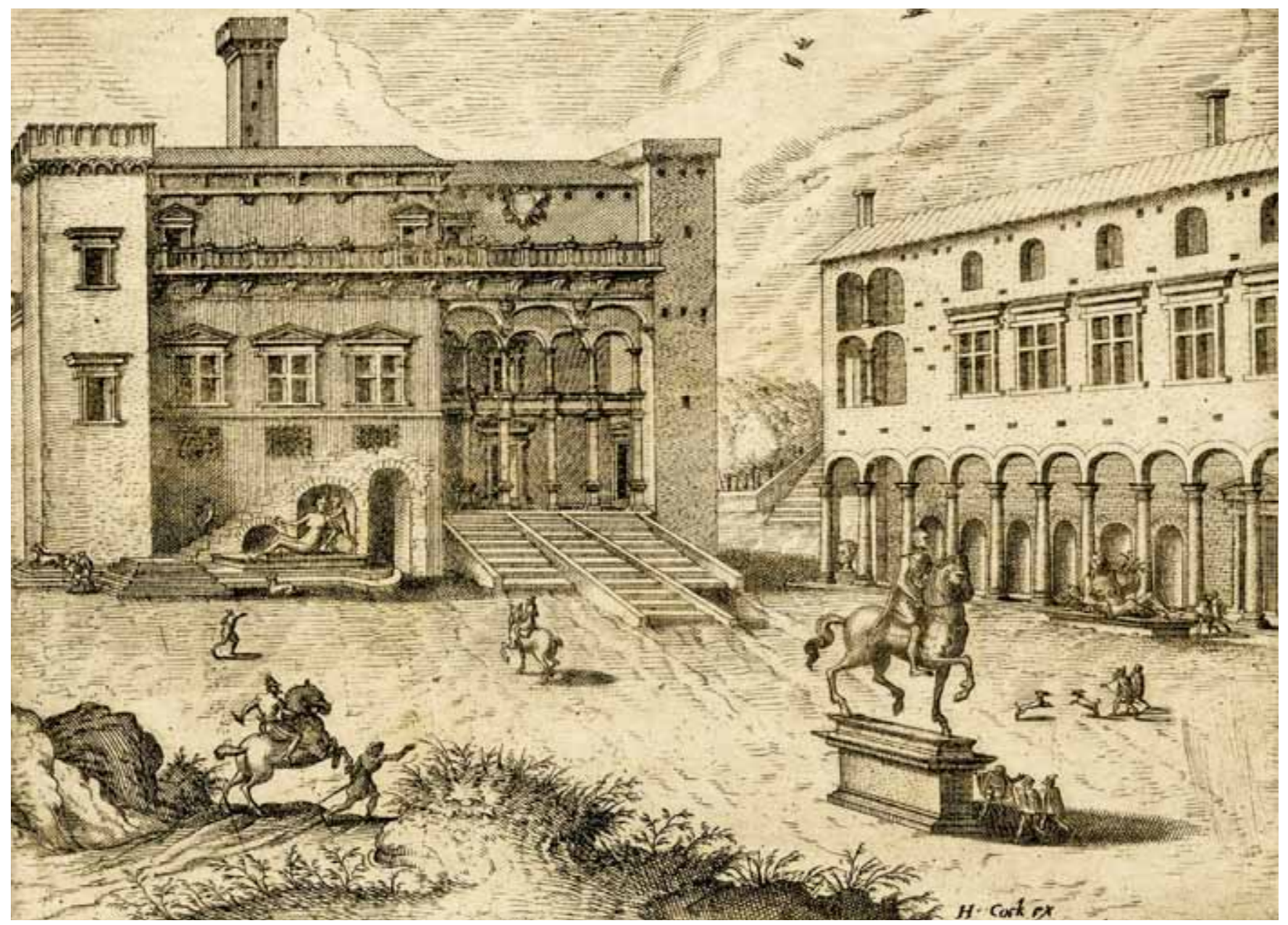

Figure I. Joannes et Lucas van Doetecum, publiée par Hieronymus Cock, Vue du Capitole, dans Operum Antiquorum Romanorum..., Anvers, Aux Quatre Vents, 1562. Gravure 15,4 × 21,I cm. Londres, The British Museum [Riggs II5; New Hollstein 254 (The Van Doetecum Family)] (photo (C) Trustees of the British Museum).

témoignage direct, les ruines romaines dans les séries de Cock sont envahies par une armée de petits artistes occupés à dessiner avec déférence les majestueuses ruines de l'ancienne capitale du monde, une habitude typiquement flamande dont la fonction rhétorique triomphe, par exemple, dans le célèbre Autoportrait au Colisée de Maarten van Heemskerck (1553 ; fig. 2 et 3) ${ }^{9}$.

Si la qualité de "document " donnée à la vue de Cock est donc historiquement légitimée, même si elle est fallacieuse, n'oublions pas qu'il s'agit d'abord d'un " monument "; elle offre aussi de nombreuses informations sur la manière dont un artiste flamand pouvait percevoir la topographie illustre et les œuvres d'art célèbres de Rome au milieu du XVI ${ }^{\mathrm{e}}$ siècle. En d'autres termes, la vue n'est pas seulement utile pour fournir des informations topographiques et historiques sur le site, mais peut permettre à l'historien de mettre en pratique ce que j'ai récemment appelé une " archéologie du regard "10. À ce propos, deux thèmes sont principalement évoqués par les historiens de l'art à propos des séries de ruines romaines. En premier lieu, les gravures témoigneraient de l'admiration des Flamands pour l'art italien et l'Antiquité, révérence pleinement assumée au sein de la maison d'édition de Hieronymus Cock Aux Quatre Vents, dont les principales gravures dans les années 1550-60 comptaient les copies d'œuvres italiennes célèbres, en particulier celles de Raphaël, les dessins classicisants de Frans Floris ou encore les planches d'architecture du "Vitruve flamand », Hans Vredeman de Vries ${ }^{11}$. Ces œuvres témoignaient de la prédominance du modèle classique italien dont la supériorité, explique Karel van Mander dans son introduction à la Vie de Jan van Scorel, 


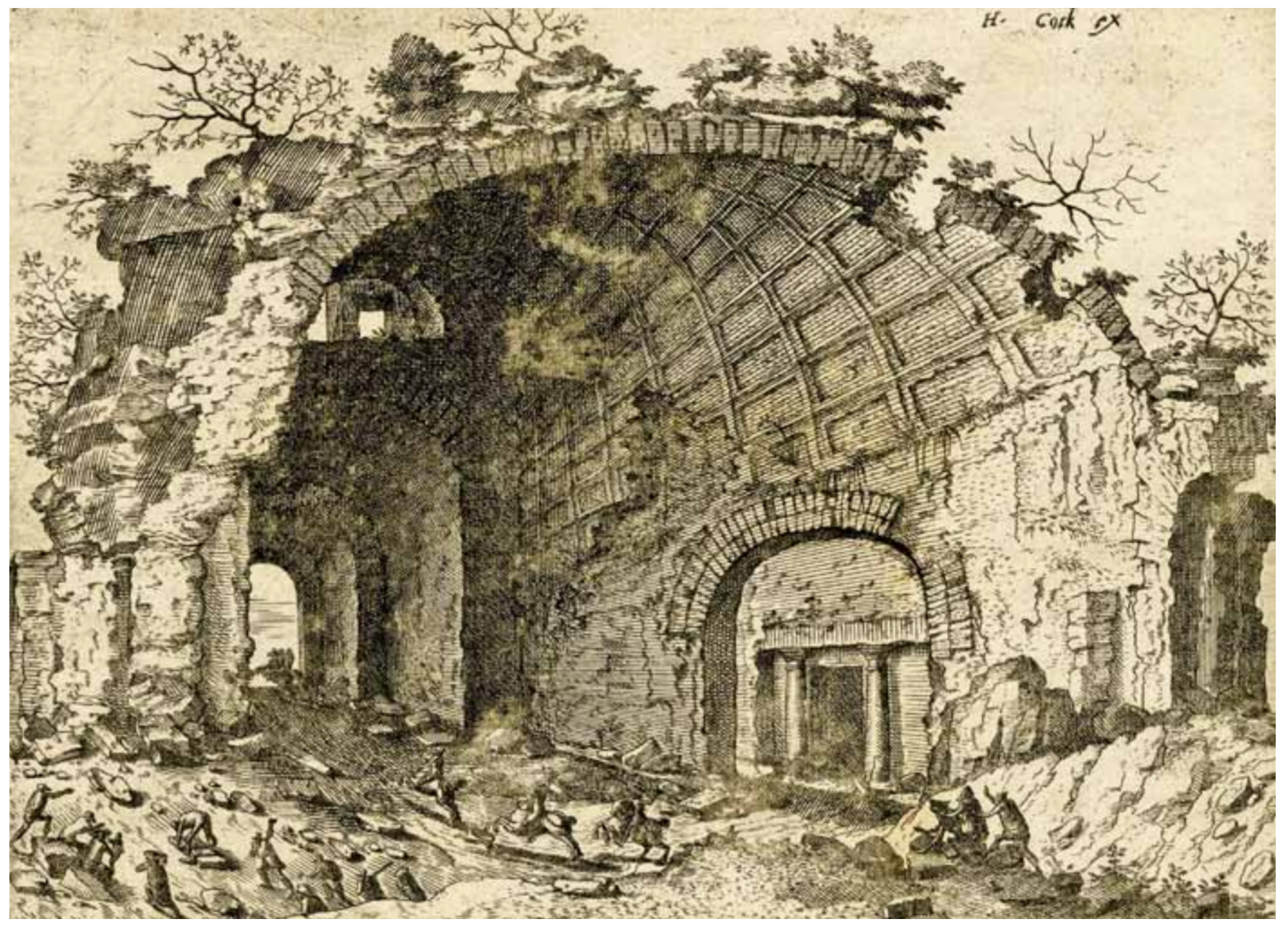

Figure 2. Joannes et Lucas van Doetecum, publiée par Hieronymus Cock, Ruine romaine, dans Operum Antiquorum Romanorum..., Anvers, Aux Quatre Vents, 1562. Gravure, 15,I × 20,8 cm. Londres, The British Museum [Riggs 122; New Hollstein 240.1 (The Van Doetecum Family)] (photo : ( ) Trustees of the British Museum).

dérivait de la présence des ruines et des œuvres de l'Antiquité offertes à la vue de tous dans les grandes collections romaines, comme celle de la place du Capitole :

On sait que Rome, l'incomparable, la reine des cités, était ornée, au temps de sa plus grande splendeur, de magnifiques statues, ou, pour mieux dire, de marbres et de bronzes qu'une suprême intelligence avait transformés en formes humaines et en figures d'animaux de la plus exquise beauté. On sait encore qu'à diverses reprises la guerre dévastatrice vint bouleverser cette noble ville, et qu'enfin, renaissant sous la pacifique domination des papes, des fouilles rendirent au jour quelques-uns de ces beaux marbres et de ces bronzes qui vinrent éclairer l'art, ouvrir les yeux de ses adeptes et leur apprendre à discerner le beau du laid par la connaissance de ce qu'il y a de plus parfait dans la création, aussi bien pour ce qui concerne la forme humaine que celle des animaux. Grâce à ce puissant secours, les Italiens ont pu arriver de bonne heure à la juste conception de la vraie nature, tandis que nos Flamands s'appliquaient encore à chercher le progrès par un travail routinier, sans autres modèles que la nature vulgaire, restant, en quelque sorte, plongés dans les ténèbres ou tout au moins, médiocrement éclairés jusqu'au jour où Jean van Schoorel rapporta d'Italie, pour les leur mettre sous les yeux, les formes les plus parfaites de notre $\operatorname{art}^{12}$.

Dans la littérature artistique, l'autre thème dominant concernant les vues de Cock est évidemment celui des ruines. 


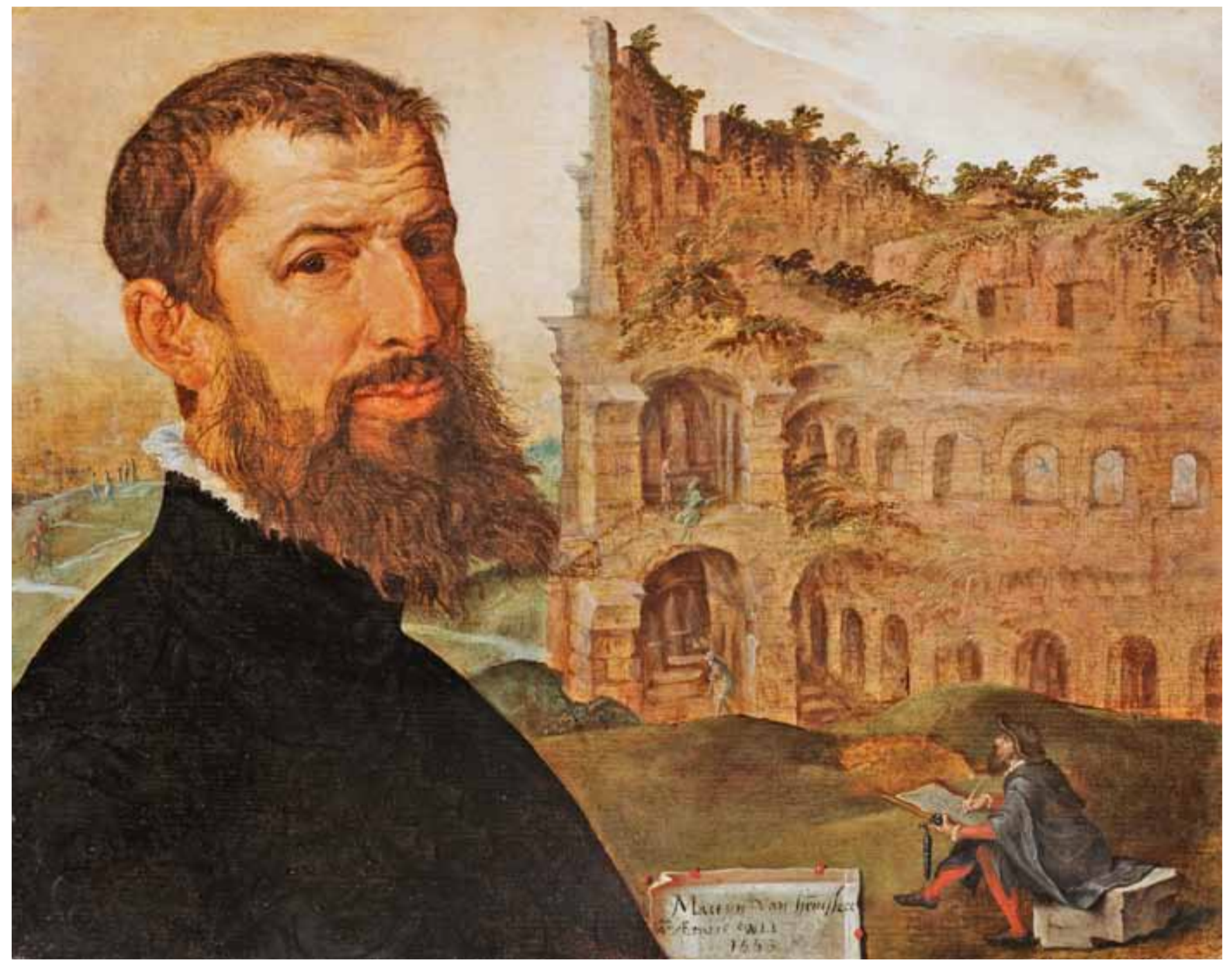

Figure 3. Maarten van Heemskerck, Autoportrait au Colisée, 1553. Huile sur panneau, 42,2 ×54 cm. Cambridge, Fitzwilliam Museum (photo : @ Fitzwilliam Museum, University of Cambridge).

Les deux thèmes étaient d'ailleurs imbriqués, l'architecture italienne de la Renaissance étant vue par les Flamands à travers le prisme des ruines de l'Antiquité. Dans les dessins de Maarten van Heemskerck ou encore dans la série gravée de Cock de 1561, l'abside inachevée de la basilique Saint-Pierre était, par exemple, clairement assimilée à une ruine antique ${ }^{13}$. Sur les traces de Jakob Burckhardt, les historiens ont amplement souligné la fonction de ces gravures et dessins de ruines, images élégiaques d'une gloire passée et symboles de la vanité des préoccupations humaines ${ }^{14}$. Ainsi, le quatrain de Cornelis Grapheus sur la page de titre de la série de 1551 lamente la destinée de Rome, " la Reine du Monde ", réduite à la ruine par la furie des barbares et le passage du temps ${ }^{15}$. Dans un article récent, Christopher
Heuer a élaboré une lecture nouvelle et stimulante des vues de Cock, basée sur l'idée d'une "esthétique de l'effondrement " (aesthetic of collapse), qui s'intègre pleinement dans cette veine poétique de l'interprétation des ruines ${ }^{16}$.

La vue du Capitole et les gravures de Cock, en particulier la série de 1562, comportent cependant une dimension qui est passée, semble-t-il, entièrement inaperçue : celle de l'humour, de la satire, du renversement ironique du sens, à l'opposé de la nostalgie méditative du Tempus edax rerum ou de l'éloge de la "vraie nature » atteinte par l'art des Anciens et ressuscitée par les Italiens. Pour voir et comprendre cette dimension, il faut poser sur ces œuvres un regard nouveau, regard rapproché, scrutateur, qui est différent du regard englobant, celui du " premier coup 
d'œil » attiré par le « sujet principal » des gravures, c'est-à-dire l'architecture et les ruines ${ }^{17}$. Car les gravures romaines de Cock nécessitent une opération du regard en deux temps, deux " moments ", et le second moment, celui du regard qui pénètre dans les méandres des ruines, qui fouille dans les coins des places, qui s'arrête sur les innombrables personnages qui les peuplent, a été systématiquement oublié par l'histoire de l'art.

La vue est construite à partir d'une diagonale qui part du coin inférieur gauche de la gravure et que matérialisent le cavalier et le personnage arrivant sur la place (fig. 1). Cette composition est caractéristique des premières vues du Capitole. Elle est très proche, par exemple, de la vue peinte vers 1543 qui orne la frise de la salle des Aigles (sala delle Aquile) du palais des Conservateurs ${ }^{18}$. Ce parti pris compositionnel met en valeur les façades des deux palais où s'exerçait le pouvoir civique de la commune de Rome, ainsi que les statues antiques qui en devinrent les symboles les plus importants à la Renaissance. Le projet de rénovation de la place par Michel-Ange pour le pape Paul III (1534-49), en créant un espace régulier sur les trois côtés, aura comme conséquence importante de focaliser l'attention sur la statue équestre de Marc Aurèle, au centre de la place ; quasi toutes les représentations du Capitole après le milieu du XVI ${ }^{e}$ siècle adopteront ce nouveau point de vue « théâtral » et " impérial ", avec le palais Sénatorial rénové vu de face et le palais des Conservateurs relégué « en coulisse " sur la droite (fig. 9). Mais la composition de Cock permet surtout de mettre en valeur ce que je serais tenté d'appeler « l'épisode principal » de la représentation, c'est-à-dire la séparation temporaire et le déplacement des statues des dieux-fleuves, le Nil et le Tibre, devant la façade du palais Sénatorial. Les deux statues, transportées depuis le Quirinal sous le pontificat de Léon X (1513-21), marquaient majestueusement l'entrée principale du palais des Conservateurs depuis 1517, comme on le voit encore dans les dessins de Maarten van Heemskerck ou ceux de Francisco de Holanda ${ }^{19}$. Lucio Fauno, dans la première édition de ses Antichità della città di Roma publiée à Rome en 1548 les mentionne encore à cet endroit, mais ajoute dans son édition de 1553, «Aujourd'hui, ces deux statues de dieux-fleuves sont en train d'être déplacées de ce lieu pour être mises devant le palais du Capitole et ils en ont déjà enlevé une "20. La séparation des deux statues a été figurée de manière hautement comique par le Flamand, au prix d'une manipulation évidente de la réalité. Le Tibre, resté seul devant le palais des Conservateurs, tourne la tête vers son compagnon désormais éloigné, qui lui rend son regard. Pour créer cet échange, Cock a modifié l'orientation de la tête du dieu de 180 degrés, une pose qui rappelle d'ailleurs celle d'une autre célèbre statue du Tibre alors conservée dans la cour du Belvédère au Vatican ${ }^{21}$ (fig. 4a et 4b). Quant au Nil, le voilà carrément travesti en femme, et la sphinge sur laquelle il s'appuie, transformée en enfant

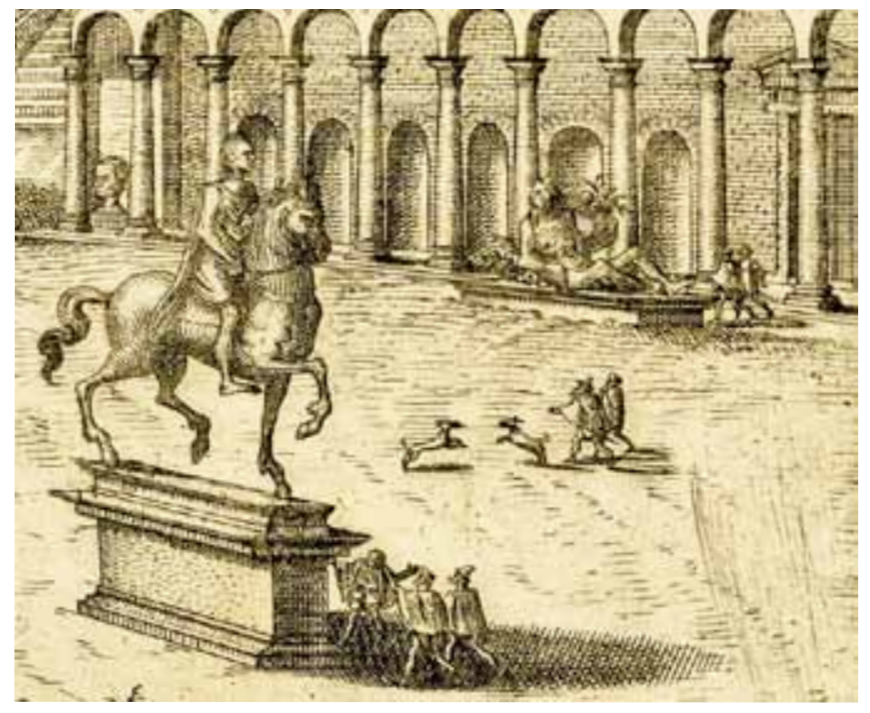

Figure 4a. Statue du Tibre dans la gravure de Cock, détail de la figure I.

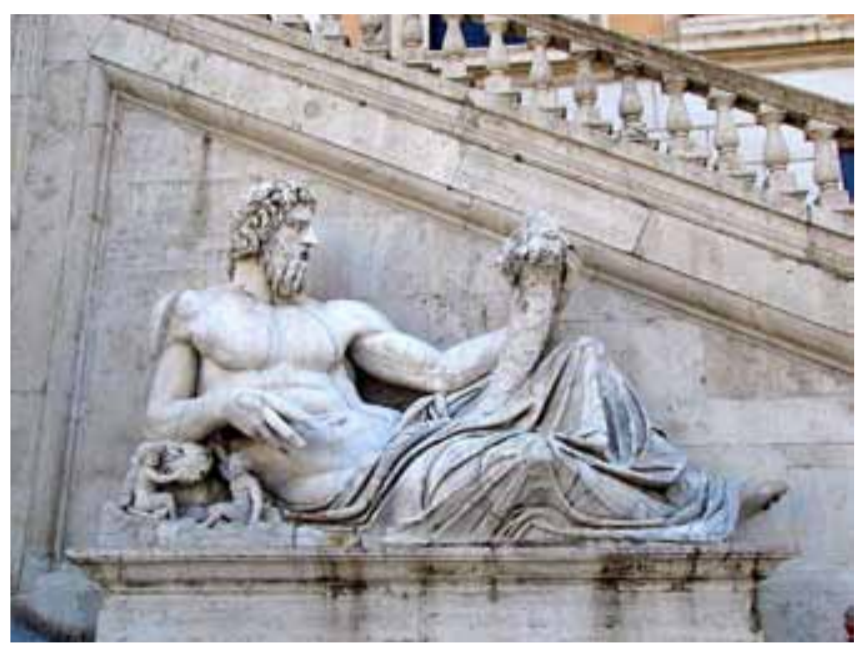

Figure 4b. Le Tibre (Tigris) de la Place du Capitole, situé sur le Quirinal depuis l'époque antique, transféré en 1517. Sculpture romaine, fin de l'époque trajane (98-II7 apr. J.-C.). Marbre blanc, h. $312 \mathrm{~cm}, I .490 \mathrm{~cm}$ (inv. 3246) (photo : auteur).

accroché à sa mère. Corne d'abondance, gouvernail, barbe et toge ont disparu par la même occasion (fig. $5 \mathrm{a}$ et $5 \mathrm{~b}$ ). Cock a transformé les deux fleuves virils, symboles par excellence de la puissance de la nature et de la perfection de l'art antique, en une touchante scène domestique : un vieux papa malheureux pressé d'aller retrouver sa femme et son enfant !

Ce commentaire burlesque sur la séparation des statues repose sans doute sur la mode qui consistait alors à grouper les statues de dieux-fleuves par paires. Dans la cour du Belvédère, 


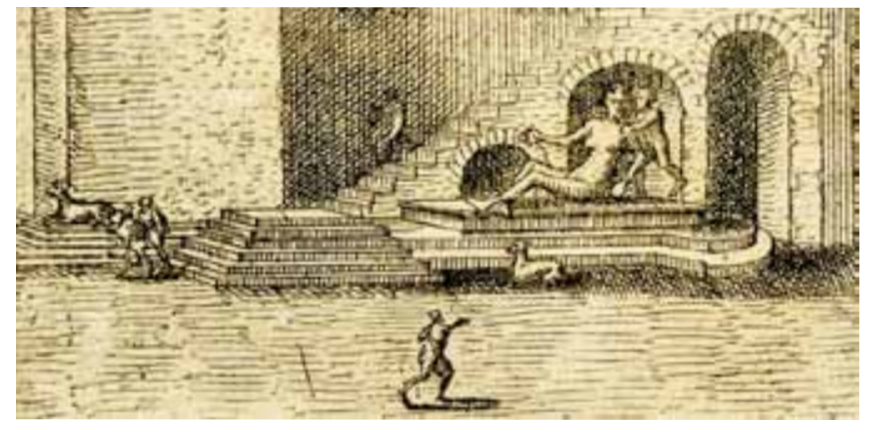

Figure 5a. Statue du Nil dans la gravure de Cock, détail de la figure I. comme à l'entrée du palais des Conservateurs, les statues du Nil et du Tibre se faisaient face et, pour ainsi dire, se regardaient, comme on le voit bien dans les dessins que Maarten van Heemskerck réalisa des deux groupes lors de son séjour romain entre 1532 et 1537. Un autre Flamand, Hermannus Posthumus, dans son tableau désormais célèbre Tempus edax rerum de 1536, plaça au centre de la composition un artiste en train de dessiner les deux recubantes du Capitole, disposés l'un en face de l'autre ${ }^{22}$. $\mathrm{Au}$ Belvédère encore, une statue du fleuve Tigris fut associée à celle de l'Ariane endormie, souvent identifiée avec Cléopâtre mourante ${ }^{23}$. Plus tard, dans la cour du nymphée de la villa Giulia ou dans les jardins de la villa Lante à Bagnaia, les personnifications du Tibre et de l'Arno seront disposées symétriquement, sur le modèle des dieux-fleuves du Capitole.

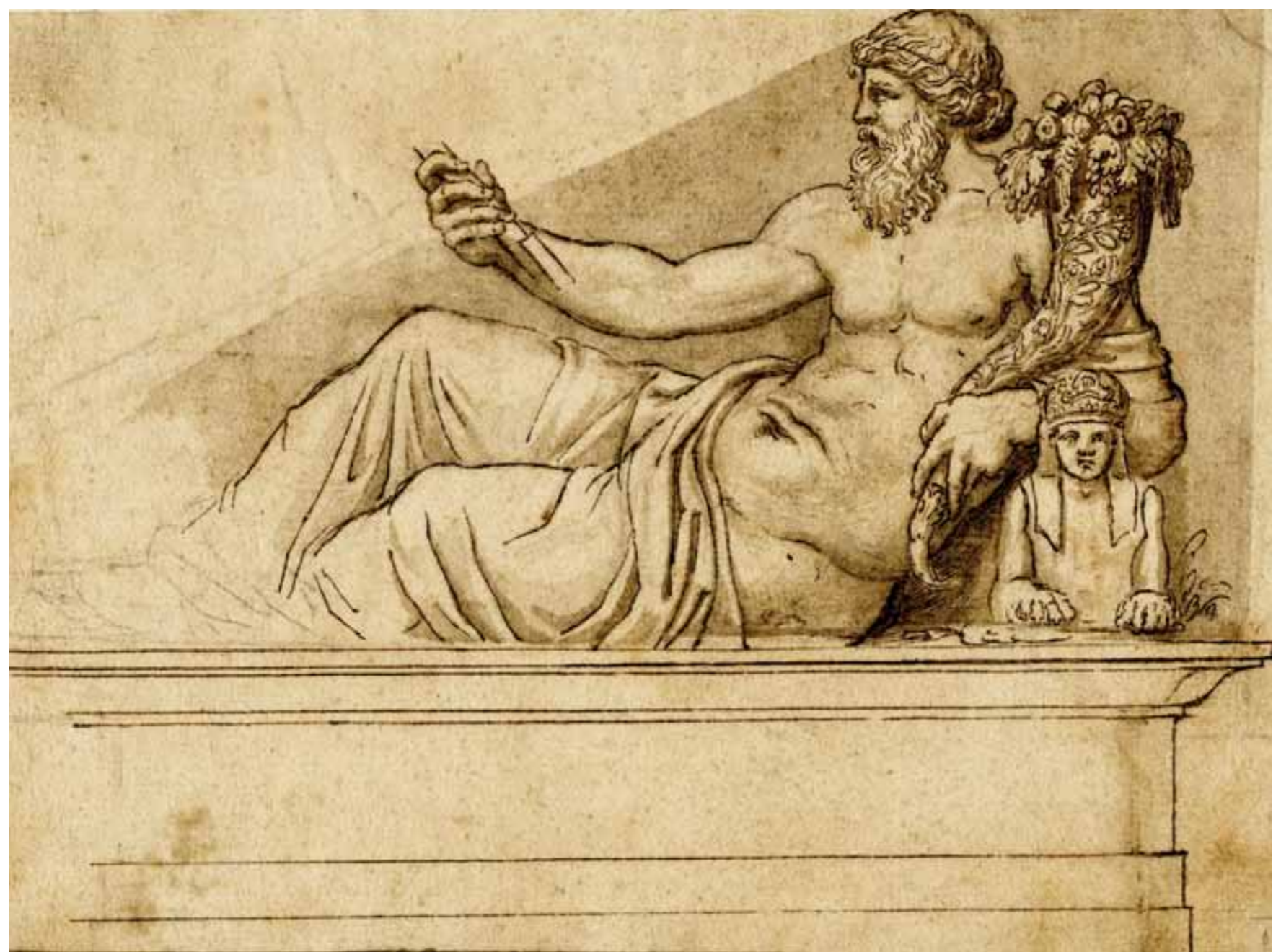

Figure 5b. Anonyme italien, Le Nil de la Place du Capitole, XVle siècle. Dessin, plume et encre brune, lavis et fusain, $14,9 \times 20, I \mathrm{~cm}$. Londres, The British Museum (photo : (c) Trustees of the British Museum). 


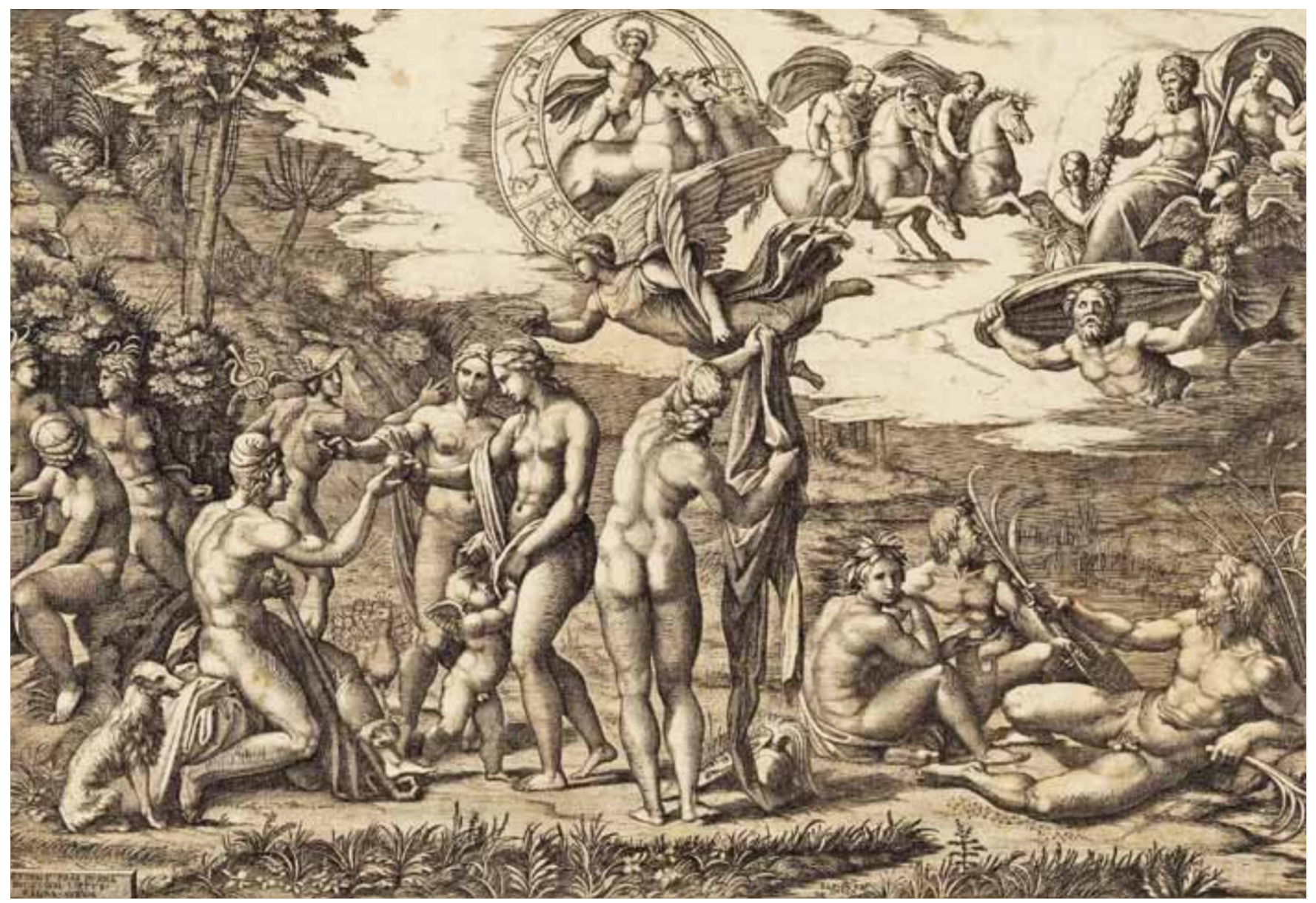

Figure 6. Marcantonio Raimondi, d'après Raphael, Le jugement de Pâris, v. 15।7-20. Gravure, 29,5 × 43,8 cm. Londres, The British Museum [Bartsch XIV.197.245] (photo : @ Trustees of the British Museum).

Si cette dimension comique, digne d'une scène de genre flamande, est unique, le fait de changer la signification des dieux-fleuves antiques à travers des installations spécifiques, des restaurations, ou des travestissements était une pratique courante dans l'Italie du XVI e siècle, comme l'a récemment réaffirmé Claudia Lazzaro24. De même, Una Roman d'Elia note combien, dans de nombreux cas, ces " restaurations " modifièrent le caractère calme et majestueux des statues antiques en leur donnant une dimension mélancolique empreinte de souffrance et de nostalgie, un pathos qu'elles ne possédaient pas à l'origine. La restauration de la statue du Tigris-Arno au Belvédère, à laquelle Michel-Ange fut associé, est un exemple symptomatique de cette " réinterprétation " de l'Antique à la Renaissance 25 . En d'autres termes, en travestissant le groupe des dieux-fleuves et en lui donnant une dimension proprement narrative, Cock faisait preuve de la même créativité que les artistes italiens, dont par exemple Michel-Ange. Son interprétation comique, voire moqueuse et satirique, manquait toutefois de la dose de décorum attendue pour de tels monuments glorieux de l'Antiquité censés, selon la formule de Van Mander citée plus haut, apprendre aux artistes - et aux Flamands en particulier "à discerner le beau du laid par la connaissance de ce qu'il y a de plus parfait dans la création ».

Malgré le traitement irrévérencieux infligé aux dieux-fleuves du Capitole, la gravure révèle un degré d'inventivité et d'expérimentation vis-à-vis de l'Antique que Cock aurait pu effectivement apprendre des grands maîtres italiens. Dans le Jugement de Pâris de Marcantonio Raimondi d'après Raphaël, une des plus fameuses gravures de la Renaissance italienne, un groupe composé d'une nymphe et de deux divinités fluviales occupe, au premier plan á droite, une place importante dans la composition (v. 1517-20 ; fig. 6). Célèbre pour avoir inspiré le Déjeuner sur l'herbe d'Édouard Manet, ce groupe n'appartient pas à proprement parler à l'historia, mais incarne plutôt le locus de la 


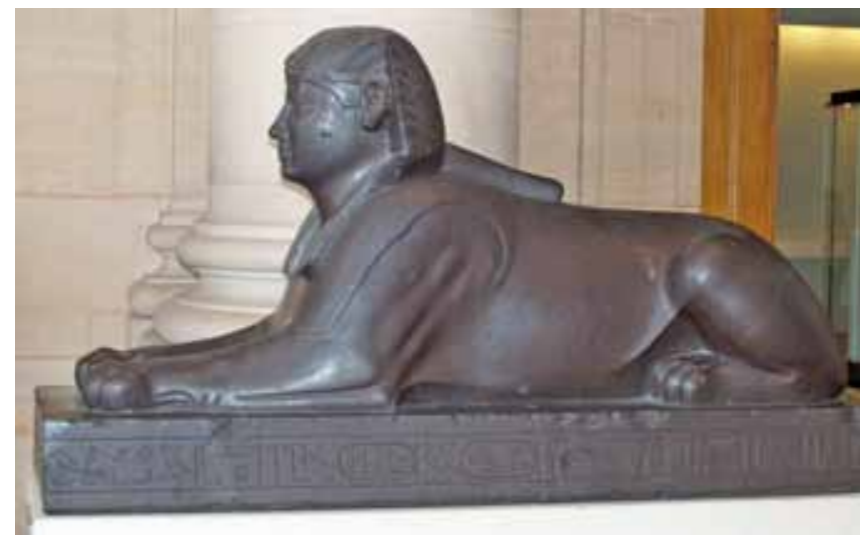

Figure 7. Le Sphinx royal au nom du pharaon Hakoris (393-80 av. J.-C.), Rome, peut-être l'Iseum du champ de Mars, puis la place du Capitole, puis la villa Borghèse. Basalte, h. $78,5 \mathrm{~cm}, \mathrm{I} .15 \mathrm{I} \mathrm{cm}, \mathrm{p} .44 \mathrm{~cm}$. Paris, Musée du Louvre (photo : auteur). rencontre entre le berger Pâris et les trois déesses Junon, Vénus et Minerve près du mont $\mathrm{Ida}^{26}$. Malgré cela, Raphaël, inspiré par des exemples identifiables de statues antiques, a animé ses figures, un peu comme le fera Cock plus tard dans sa gravure du Capitole, en construisant un réseau dense de regards à l'intérieur de l'espace de la représentation, donnant ainsi une sorte de narrativité intrinsèque à l'image.

Cock, cependant, ne s'arrête pas aux statues des dieuxfleuves. Ce qui apparaît dans la gravure comme une paire de braves toutous sagement assis au pied des escaliers et de la statue du Nil sont en fait deux célèbres statues de sphinges égyptiennes installées sur le Capitole à l'époque de Léon X et documentées pour la première fois au pied de la rampe du palais Sénatorial dans l'Antiquaria Urbis d'Andrea Fulvio publié en 1513 (p. 39) (fig. 5a et 7). Aujourd'hui au Louvre, elles furent déplacées vers 1547 à des endroits séparés sur la place du Capitole et dans le

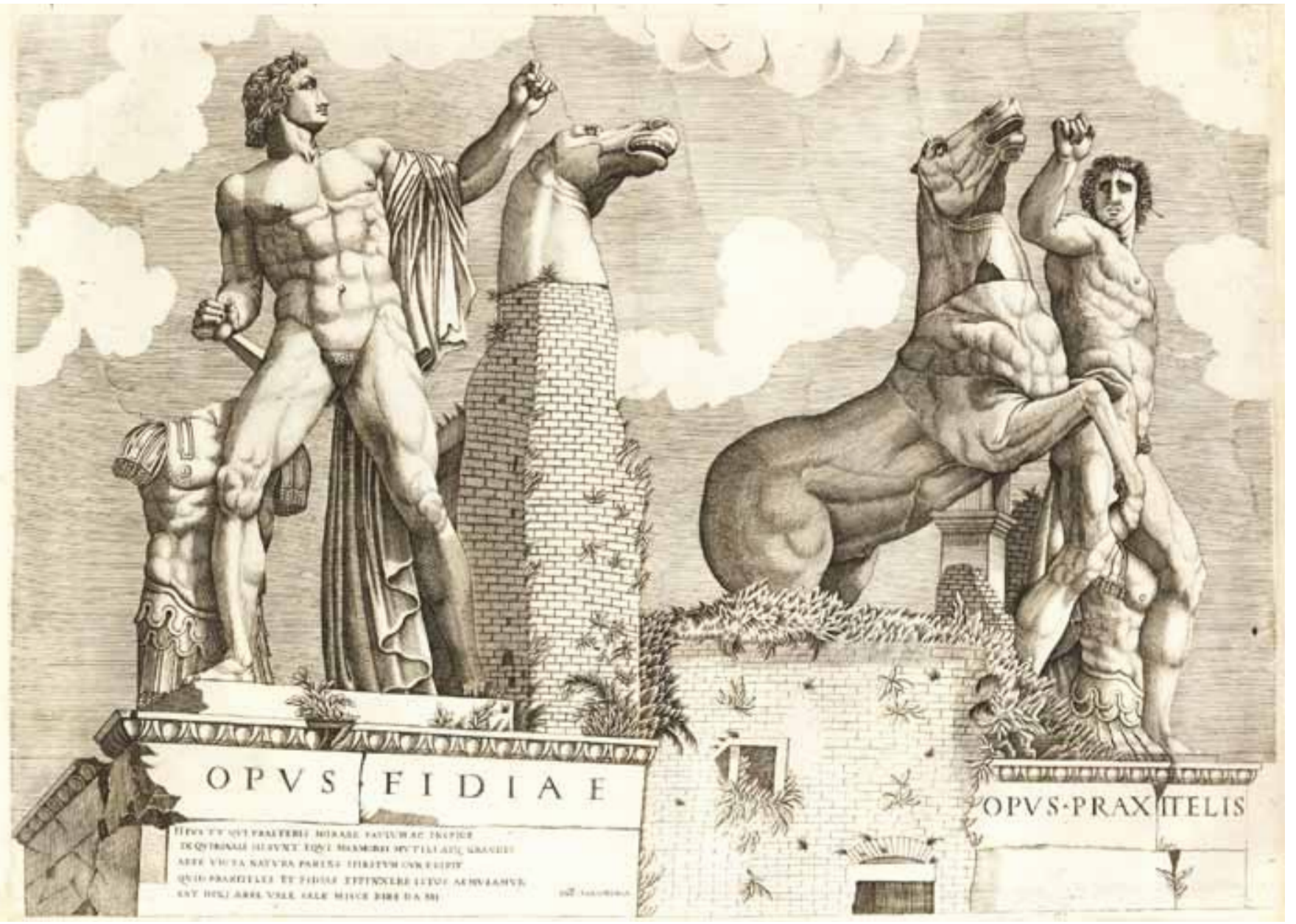

Figure 8. Anonyme, publiée par Antonio Salamanca, Les Dioscures sur la piazza Montecavallo (Opus Fidiae Opus Praxitelis), dans Speculum Romanae Magnificentiae, 1538-46. Gravure, 36,9 × 50,4 cm. Londres, The British Museum (photo : (C Trustees of the British Museum). 


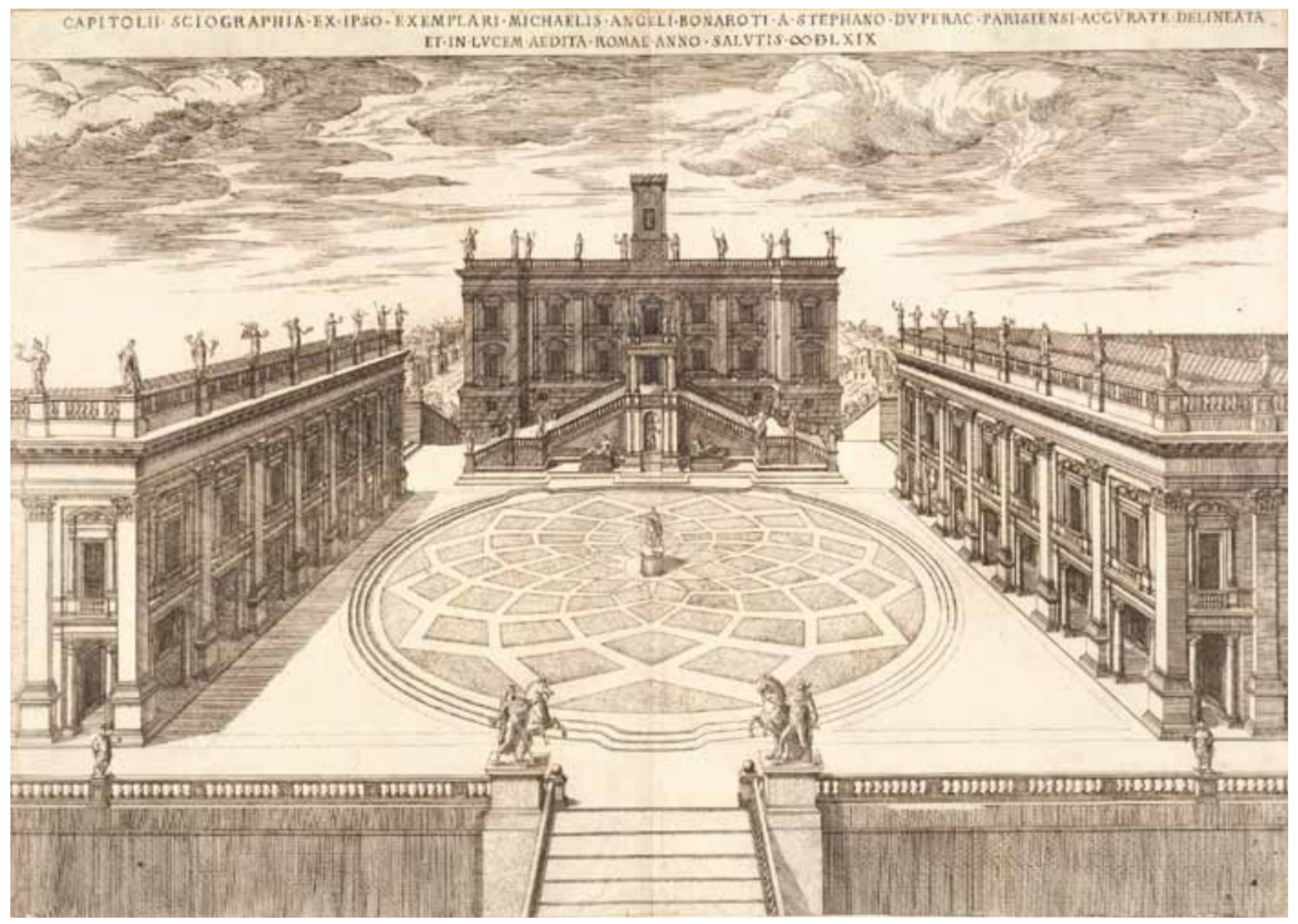

Figure 9. Étienne Dupérac, La place du Capitole d'après le dessin de Michel-Ange, 1569. Gravure, 37,1 ×54cm. Londres, The British Museum (photo : () Trustees of the British Museum).

palais des Conservateurs avant d'être transférées à la Villa Borghèse27. Leur présence sur la célèbre colline en fit bien vite des symboles " civiques " de la civilisation romaine et inspira de nombreux artistes, parmi lesquels Jules Romain ${ }^{28}$.

Même la statue équestre de Marc Aurèle reçut de la part de Cock un traitement un peu spécial (fig. 1, 4a). L'empereur, sans sa barbe, et dont le bras levé a été omis, tourne la tête vers la statue du Tibre esseulée, participant ainsi au jeu des regards que s'échangent les statues. L'idée que la célèbre sculpture ait pris vie est encore renforcée par la présence, au centre de la place, d'un visiteur à cheval tourné vers le palais Sénatorial. Situé dans l'axe du grand groupe de bronze, il en imite exactement la pose, comme en miroir. Cock réussit ainsi avec brio à élaborer un commentaire sur la statue de Marc Aurèle, dont il présente deux points de vue différents dans une seule image, référence peut-être au débat sur le Paragone qui alimentait les conversations des amateurs au milieu du XVI siècle, comme ceux que Cock a figurés dans l'image autour de chaque statue 29 .

À ce duo équestre, vient s'ajouter, sur la gauche, un troisième cavalier débouchant sur la place. À côté du cheval cabré, un personnage à pied, le bras tendu, indique la statue de Marc Aurèle. Or, ce groupe apparemment anodin de touristes constitue une citation évidente des fameux Dioscures du Quirinal. Les positions du cheval cabré et du personnage au bras levé (avec une pose inversée dans la gravure de Cock) correspondent à celles des gigantesques statues qui donnèrent leur nom au Monte Cavallo (fig. 8). La citation du groupe des Dioscures est doublement signifiante. Elle permet d'une part de confronter les deux grands types compositionnels de la statuaire équestre antique - le cheval cabré et le cheval reposant sur un membre postérieur et un autre antérieur opposé - qui constituent les 


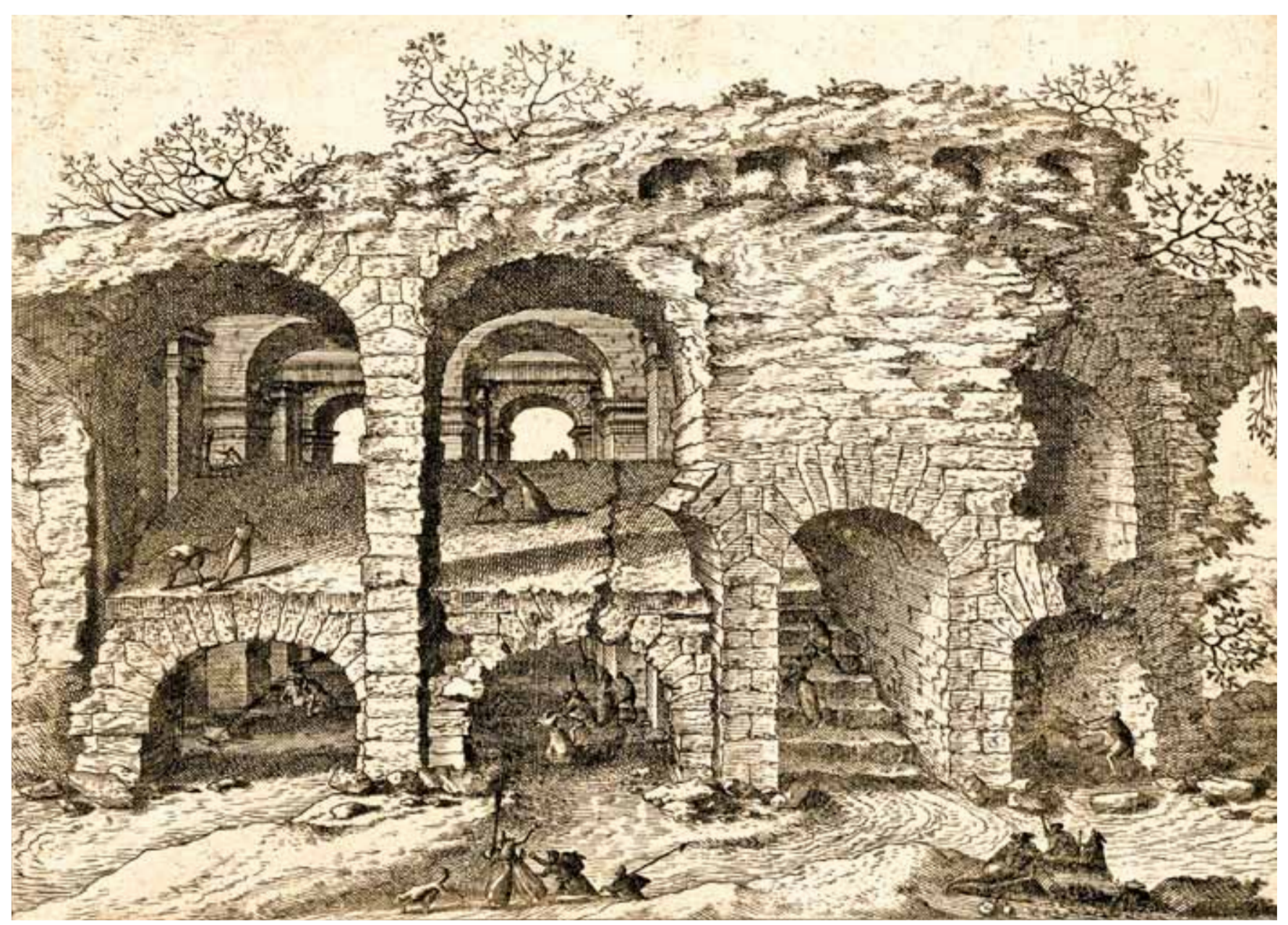

Figure 10. Joannes et Lucas van Doetecum, publiée par Hieronymus Cock, Ruine romaine (Colisée?), dans Operum Antiquorum Romanorum..., Anvers, Aux Quatre Vents, 1562. Gravure, $15 \times 20,7$ cm. Londres, The British Museum [Riggs 116 ; New Hollstein 242.1 (The Van Doetecum Family)] (photo : (C) Trustees of the British Museum).

sources principales pour les nombreux monuments et portraits équestres de la Renaissance. Que les mérites, la beauté ou la faisabilité technique de l'une ou l'autre solution, riches de multiples associations historiques et symboliques, aient pu nourrir la discussion des visiteurs est suggéré par le détail, sur la droite du groupe équestre, des deux chiens juchés sur leurs pattes arrières, cherchant, semble-t-il, à imiter la pose des célèbres chevaux antiques ${ }^{30}$ (fig. 1 et 4a)! La citation des Dioscures par Cock est également intéressante d'un point de vue historique dans la mesure où l'on sait que Paul III avait prévu installer le groupe sur la place du Capitole, et que seule l'insistance de Michel-Ange l'en avait finalement dissuadé31. Il est fort possible que Cock ait été au courant de ce projet qui sera plus tard documenté dans une célèbre gravure d'Étienne Dupérac montrant la place telle que Michel-Ange l'avait projetée (fig. 9).
En conclusion, la gravure de Cock utilise l'humour et la dérision, à l'opposé du sérieux et du décorum qui caractérisent le discours artistique italien, pour commenter la célèbre collection de sculptures de la place du Capitole au milieu du XVI ${ }^{\mathrm{e}}$ siècle. Sans être immédiatement apparents, les détails cocasses et les manipulations de la réalité opèrent comme des clins d'œil à la fois amusants et riches de sens pour celui qui les repère. Une analyse même superficielle des autres gravures des séries romaines de Cock peut nous permettre de mieux comprendre l'origine et la signification probable d'un tel renversement ironique et comique du sens.

Pour le public flamand qui constituait la clientèle de Cock, les gravures de ruines n'étaient pas de simples images du paysage romain. Elles constituaient aussi un divertissement visuel et un 


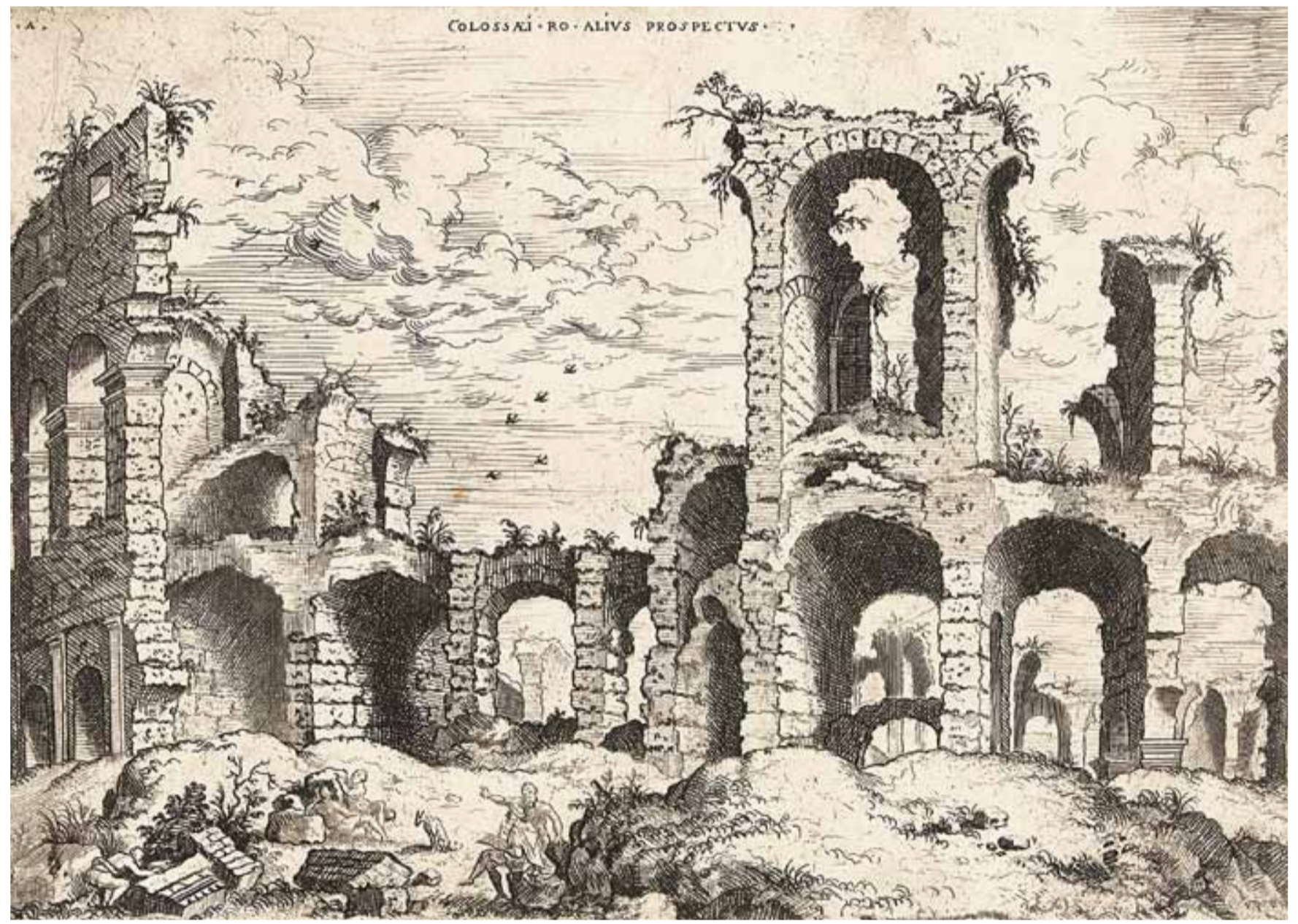

Figure II. D'après un dessin de Hieronymus Cock, publiée par Hieronymus Cock, Vue du Colisée, dans Praecipua aliquot romanae antiquitatis ruinarum monimenta..., Anvers, Aux Quatre Vents, I55I. Gravure, 23,3 × 33,7 cm. Amsterdam, Rijksmuseum [Riggs, 3 ; Hollstein Dutch 23] (photo : () Rijksmuseum, Amsterdam).

commentaire moral. On peut s'en convaincre en fouillant un peu dans les recoins des ruines. Dans une gravure du Colisée de 1562, par exemple, c'est tout un monde que Cock a figuré (fig. 10) : au premier plan, un groupe de pèlerins fatigués se repose et des soldats s'affairent. À droite, sous une voûte ruinée, c'est un homme qu'on assassine, tandis qu'un peu plus loin une femme tente d'échapper à ses agresseurs. Un autre personnage entraîne une prostituée dans un coin du théâtre. Dans l'ombre d'une voûte, on distingue un couple en train de forniquer. Ces scénettes de brigandage, de meurtres et de dépravation se retrouvent dans presque toutes les gravures et elles font d'autant plus frissonner ou sourire que l'artiste les a placées dans l'ombre, cachées pour mieux être cherchées, laissant au spectateur la liberté d'imaginer la suite... Elles n'en reflètent pas moins parfaitement la réalité historique, tout comme les duels (Riggs 128), les parties de chas- se $(99,117)$, les processions et réunions de gueux $(129,130)$, ou encore les pierriers et les carriers emportant les fragments des monuments antiques $(119,122)$ dont Cock a peuplé ses vues (fig. 2). Plus que du réalisme pittoresque, ces petites vignettes signalent surtout, me semble-t-il, une critique morale, celle des mœurs dissolues des Romains du XVI e siècle, implicitement associés à l'idée de la ruine. Que peut bien signifier, par exemple, le détail de ce personnage agenouillé en adoration devant une statue de Priape (1561 ; Riggs 99) ? Cock accuserait-il les Romains, non seulement de luxure, mais aussi d'idolâtrie païenne? Doit-on y percevoir une critique religieuse de Rome, siège de la papauté, dans l'esprit de la Réforme ? La figure du dessinateur, qui est, on l'a dit, omniprésente dans les vues de Cock, joue donc un double rôle. Elle évoque l'urgence d'enregistrer par le dessin ce qui est en train de disparaitre — littéralement — sous 
les yeux de l'artiste (la juxtaposition du dessinateur et des pilleurs de ruines dans une des gravures est éloquente à cet égard) 32 (fig. 2). Mais il est aussi observateur de la ruine de la société romaine contemporaine, un sentiment que partageront de nombreux étrangers venus visiter la "Ville éternelle " au milieu du $\mathrm{XVI}^{\mathrm{e}}$ siècle, tel Joachim du Bellay, pour ne prendre qu'un seul exemple célèbre. " Nouveau venu, qui cherches Rome en Rome / Et rien de Rome en Rome n'aperçois, / Ces vieux palais, ces vieux arcs que tu vois / Et ces vieux murs, c'est ce que Rome on nomme... " : les vers célèbres des Antiquités de Rome (1558) traduisent bien l'esthétique des gravures de Cock. Ils célèbrent certes l'âge d'or de l'Antiquité, mais représentent en même temps une théâtralisation satirique de la vie à Rome, ils suggèrent l'effacement, l'effritement, «l'effondrement » d'une image noble de la ville, la désillusion d'un regard 33 .

L'idéal de l'Antiquité reçoit donc, dans les gravures de Cock, un double éclairage. Sans prétendre que les séries des ruines n'aient pas contribué à l'éloge de l'esthétique classique, elles en donnent en tous les cas, à bien y regarder, une interprétation essentiellement subversive. La coexistence au sein des séries de ruines de la figure du dessinateur et de celle du kakker, littéralement "celui qui défèque ", est un autre symptôme de ce paradoxe, de cette tension entre le sublime et le ridicule, le noble et le vulgaire 34 (fig. 11). Figure de l'avilissement scatologique, le kakker représenté dans les ruines joue comme motif de la souillure et du ridicule de Rome, ville de la ruine et de la corruption. L'excrément, produit diabolique, fut, au XVI siècle, régulièrement associé aux controverses religieuses entre catholiques et protestants 35 . Dans les tableaux de Joachim Patinir du début du XVI siècle, le kakker est d'ailleurs souvent placé dans la zone du paysage correspondant à l'Enfer, comme dans le Paysage avec Charon ou La fuite en Égypte du Musée du Prado. Signe d'inversion de sens, de perversion diabolique, le kakker joue en même temps un rôle de divertissement comique et d'exercice du regard : dans le Schilderboeck ou Livre des peintres publié en 1604, Karel Van Mander rapporte que les amateurs de peinture (liefhebbers) de son époque pariaient entre eux à qui aurait trouvé en premier le petit personnage en train de déféquer dans les œuvres de Patinir ${ }^{36}$. Les kakker de Cock joueraient aussi ce double rôle : signes de la déchéance d'une civilisation, mais aussi détails comiques qui provoquent le regard.

Au premier rang des artistes qui firent ample usage de ce motif irrévérencieux du chieur, inspiré de la rhyparographie antique (la peinture de sujets sordides) que Pline l'Ancien oppose à la mégalographie (la peinture de sujets nobles et élevés; Histoire naturelle XXXV), figure Pieter Bruegel, lequel fut intimement lié à Hieronymus Cock et à sa maison d'édition des Quatre Vents à partir de 1555 et jusqu’à son départ pour Bruxelles en 156337. Souvent associé à sa signature, le kakker chez Bruegel peut être compris comme un double pervers de la figure de l'artiste, et le motif ordurier comme la métaphore d'une répudiation volontaire de l'idéal artistique et noble issu de l'Antique que portait aux nues la théorie de l'art de son époque. Si un tel rapprochement est apparemment incongru, on le retrouve pourtant dans les gravures de Cock, par exemple dans une vue du Colisée de 1551 (fig. 11). Au premier plan au centre, Cock a figuré un dessinateur au travail ; dans l'angle gauche, un personnage est en train de déféquer, appuyé sur un fragment de ruine. Les deux personnages qui constituent le point d'entrée du regard dans la composition offrent au spectateur deux clefs de lecture bien contradictoires. Précisément là où, sur la gauche de l'image, Maarten van Heemskerck peignit son autoportrait de 1553, en s'inspirant sans doute d'un portrait de Michel-Ange, Cock place la figure la plus vile et la plus ridicule qui soit (fig. 3). Plus tard, Bruegel installera aussi un petit chieur devant sa vue de La Tour de Babel du musée de Vienne de 1563 : pour lui, pourrait-on dire, la tour de Babel, directement inspirée par le Colisée, était au vaniteux monument romain ce que la figure du chieur était à celle du dessinateur romanisant ${ }^{38}$. Dans un article récent sur la figure du kakker dans l'art flamand des XVI et $\mathrm{XVII}{ }^{e}$ siècles, Michel Weemans a bien saisi l'importance théorique de ce motif scatologique apparemment anodin :

Chez Bruegel, [...] les détails rhyparographiques opèrent selon deux manières : leur sens est intrinsèque, il produit une tension à l'intérieur de l'image, mais il est aussi extrinsèque à l'image, opposé à un modèle dont il inverse et parodie les valeurs. [...] Chez Bruegel le rhyparographe est brandi contre le courant romanisant qui, à partir des années 1550, s'efforce de s'imposer comme modèle dominant, témoin de l'engouement des Fiamminghi pour l'art italien. La transgression rhyparographique chez Bruegel est l'ouverture d'une mêlée critique dont quelques textes reflètent la virulence, et dont le lieu fut la peinture, et d'abord la gravure 39 .

La «mêlée critique » dont parle Weemans fait notamment référence à un poème contemporain de Lucas de Heere célébrant la fameuse série des Arts libéraux de Frans Floris, mais qui se veut aussi une "invective adressée à un certain peintre " qu'il n'est pas difficile d'identifier avec Bruegel. Le poème oppose deux modèles d'artistes : l'artiste cultivé et l'artiste inculte, celui qui se rattache au canon classique et celui qui l'ignore. L'un est loué pour ses qualités de "grâce ", de " décorum » et de "maniera »; l'autre n'est qu'un "onghemanier ", un artiste sans manière, un peintre de "kaermespoeppen ", de " poupées de kermesses " 40 . Ces deux courants antagonistes coexistaient au sein de la maison d'édition de Cock depuis l'arrivée de Bruegel. Aux œuvres italianisantes d'un Frans Floris, à la rigueur géométrique classicisante d'un Hans Vredeman de Vries, Bruegel avait répondu en apportant à Cock, à son retour d'Italie, une série de dessins de 


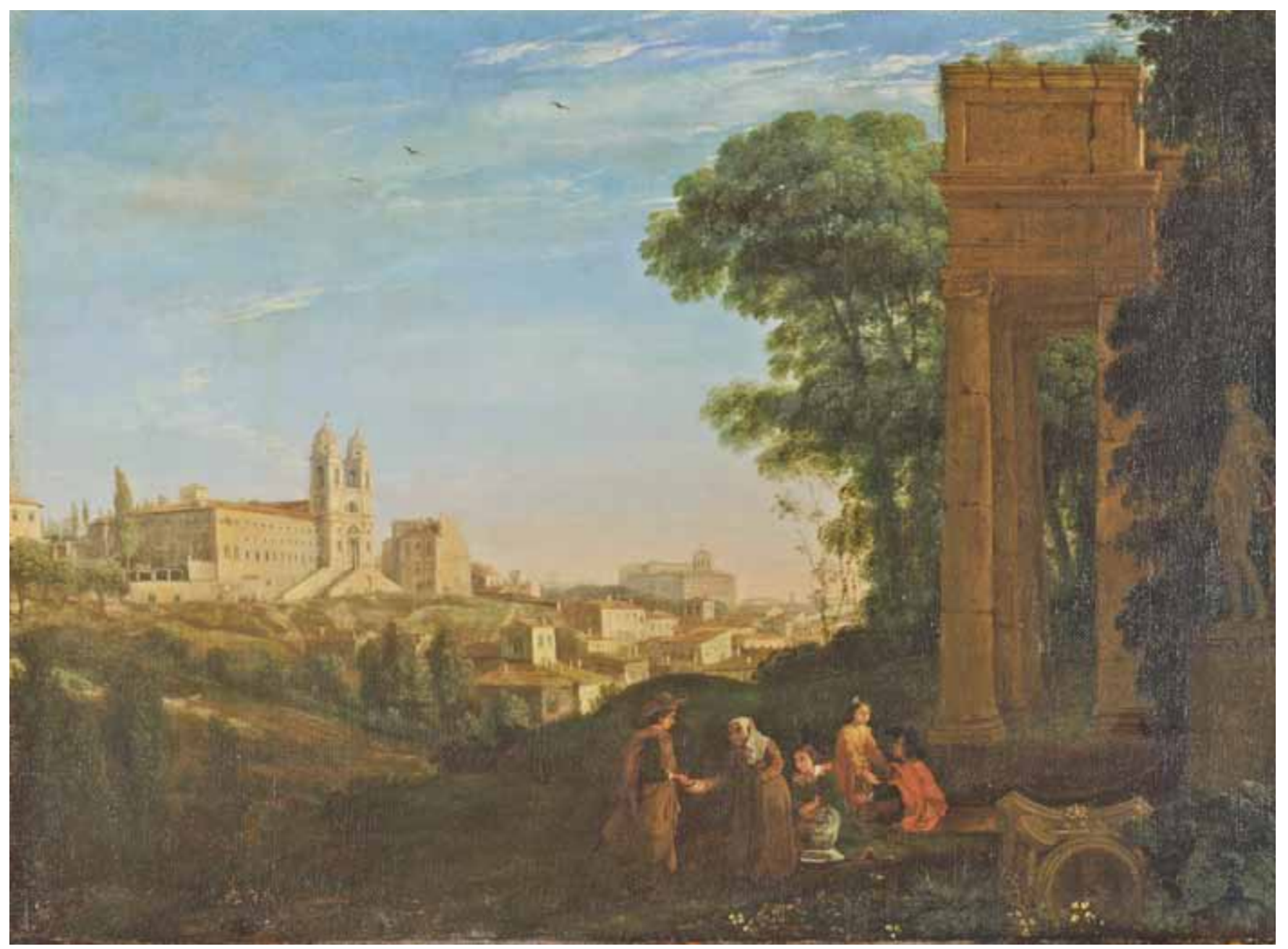

Figure 12. Claude Lorrain, Vue de la Trinité-des-Monts à Rome, 1632. Huile sur toile, 60,3 × $84 \mathrm{~cm}$. Londres, National Gallery (photo : @ National Gallery Picture Library).

paysages qui modernisait la tradition du Weltlandschaft inaugurée par Patinir, la célèbre série des Grands paysages, mais aussi des compositions grotesques et burlesques inspirées de Hieronymus Bosch ; d'un côté donc, l'adéquation au modèle artistique et théorique italien, de l'autre, des sujets qui lui étaient précisément opposés ou marginaux, emblématiques de la tradition de la peinture flamande ${ }^{41}$. À cet égard, il est symptomatique que, pour parler de la fameuse série des Grands paysages publiée par Cock, Van Mander reprenne la métaphore de la souillure et de la déjection, adoptant ainsi le même procédé de renversement satirique qui caractérise la production bruegelienne : "On a dit que pendant son séjour dans les Alpes, il avait avalé tous les monts et rochers, pour au retour chez lui les vomir sur ses toiles et ses panneaux, tant il parvenait à rendre la nature avec fidélité »42. La peinture de paysage flamand serait ainsi, chez Van
Mander, présentée à travers l'imaginaire des scènes de beuveries des kermesses paysannes avec lesquelles Bruegel fut irrémédiablement associé 43 .

Les séries de ruines de Cock, en particulier celle de 1562, viendraient donc se placer au croisement de deux modèles. Si les sujets des gravures renvoient à la tradition d'exaltation de l'architecture antique et à la dimension élégiaque de sa ruine, l'insertion sous des arches écroulées et des " ruines poussiéreuses " d'un monde corrompu et avili, sorte de figuration archétypique de l'Enfer (on pense bien sûr à la série des Sept péchés capitaux de Bruegel que Cock va graver en 1558), est profondément redevable de l'univers comique et satirique de Pieter Bruegel. La dimension "pittoresque " et " paysagère » des ruines de Cock, si différente des gravures sur le même sujet publiées en Italie par Antoine Lafréry et Antonio Salamanca, est un autre symptôme 
de cette opposition entre "l'espace architectural incarnant la conception romanisante et sa subversion par le paysage " qui marque l'œuvre de Bruegel 44 . Van Mander en donne encore un autre exemple qui, s'il est sans doute inventé, n'en est pas moins emblématique :

[Hans Vredeman de Vries] obtint [...] la commande d'une peinture représentant un pavillon d'été en perspective et y introduisit, entre autres, une porte ouverte, dans l'embrasure de laquelle Pierre Brueghel, trouvant par hasard l'attirail du peintre, avait représenté un paysan, la chemise non immaculée, fort avant dans les bonnes grâces d'une paysanne, groupe qui fit joliment rire et plut considérablement au maître de la maison, qui ne voulut, à aucun prix, le laisser effacer ${ }^{45}$.

L'humour de Bruegel — surnommé " Pierre le Drôle " 46 devait être pleinement partagé par Cock, tout comme il l'était, semble-t-il, du public flamand qui s'en délectait. Van Mander retient en effet que Cock " était humoristique " et pratiquait les jeux de mots, et souligne son association avec les « rhétoriqueurs " (rederijkers $)^{47}$.

Les manipulations et travestissements des statues du Capitole dans la gravure de Cock de 1562 doivent donc se comprendre au sein d'un large mouvement culturel et artistique qui vit, au milieu du XVI ${ }^{e}$ siècle, les artistes flamands réagir face aux modèles antiques et italiens. Cette attitude fut sans aucun doute exacerbée par l'antagonisme religieux croissant entre la culture réformée du nord de l'Europe et celle de la Rome des papes, lesquels n'hésitaient pas à identifier leur pouvoir avec celui des empereurs de l'Antiquité, grâce notamment à leurs splendides collections de statues. Les grandes collections romaines comme celles de la cour du Belvédère ou de la place du Capitole, intimement associées au pouvoir papal, se trouvaient au cœur d'un débat de nature théorico-artistique qu'il est difficile de dissocier entièrement de la dispute religieuse entre protestants et catholiques ${ }^{48}$. Les images grotesques de paysans de Bruegel ou de Pieter Aertsen, inspirées curieusement par l'art italien et la statuaire classique, sont typiques de ce renversement paradoxal de la dignité artistique italienne - Reindert Falkenburg développe la notion de "paradoxical encomium " pour qualifier cette attitude comme une réponse humoristique et incisive à la supériorité de ceux qui considéraient les artistes transalpins comme n'ayant de talents que dans les mains ${ }^{49}$. En associant aux "sujets sordides " de la rhyparographie, des figures et des motifs nobles qui convenaient à la « mégalographie » tant estimée des Italiens, les Flamands se gaussaient ; si l'art de l'Antiquité demeurait une référence fondamentale dans leurs œuvres, ils répondaient à la théorie de l'art italienne qui les avait mis sur la touche par ce qui était pratiquement devenu des manifestes nationaux : le rire, l'excrément et le vomi. C'est sans doute Albrecht Dürer qui réagit le premier à l'arrogance italienne en satirisant l'art antique dans ses gravures de paysans, comme l'a récemment soutenu Jürgen Müller ${ }^{50}$. Mais cette tension entre, d'une part, la fascination pour l'art antique et le paysage de Rome et, d'autre part, sa perversion comique et satirique, est également typique des œuvres flamandes produites à Rome au XVII e siècle, notamment par Pieter van Laer et les Bamboccianti51. Cette dimension est moins souvent évoquée dans l'œuvre de Claude Lorrain, sujet tabou sans doute lorsqu'il s'agit de "l'inventeur " du paysage idéal et classique, bien que sa proximité avec les Bentvueghels dans les années 1630 soit désormais bien attestée52. Dans certaines de ses œuvres, comme dans celles des Bamboccianti, on retrouve la figure du dessinateur, mais aussi, plus inattendu, l'insolent kakker, de même que tout le vulgus que l'on a rencontré dans les gravures de Cock $^{53}$. Dans la célèbre Vue de la Trinité des Monts de la National Gallery de Londres (fig. 12), le peintre a juxtaposé de majestueuses colonnes antiques et une statue d'Apollon à la lyre avec, il faut bien l'admettre, une scène de prostitution négociée par une vieille entremetteuse. Réalisme topographique, éloge de l'Antique et renversement satirique : tous les ingrédients qui, nous l'avons vu, marquent la production des gravures de ruines de Hieronymus Cock sont à l'œuvre dans le tableau du Lorrain. Comme chez Dürer, Bruegel et Cock sans doute, il faut lire cette inversion - comique ou effrontée selon le point de vue - comme une pique adressée à cette communauté artistique italienne qui traitait les étrangers venus du Nord avec autant de méfiance que de condescendance : à cet égard, la vue de Claude "regarderait " donc moins l'Église de la Trinité des Monts que l'adjacent Palazzo Zuccari, siège de l'Académie de Saint-Luc, gardienne de l'autorité de la peinture italienne, c'est-à-dire de la seule " bonne peinture " avait dit Michel-Ange, académie où l'on se moquait bien des capacités artistiques des fiamminghi de Rome54. Rira bien qui rira le dernier...

\section{Dédicace}

Pour Marcello Fagiolo, à l'occasion de son soixante-dixième anniversaire.

\section{Notes}

Cette recherche a bénéficié du soutien financier du F.Q.R.S.C. dans le cadre d'un projet de recherche en cours (2011-2014) sur « la figure du dessinateur dans le paysage de Dürer à Claude Lorrain ».

À moins d'indication contraire, les traductions sont celles de l'auteur.

1 Herbert Siebenhuber, Das Kapitol in Rom, Idee und Gestalt, Munich, Kösel-Verlag, 1954 et James Ackerman, "Review of Siebenhuber, 1954 ", The Art Bulletin, vol. 38, no 1, mars 1956, p. 53-7, p. 55 datent la vue de 1547 d'après les documents d'archives. Après eux, un nombre important d'historiens font de 
même. Adolf Michaelis, "Michelangelos Plan zum Kapitol und seine Ausführung ", Zeitschrift für Bildende Kunst, N.F. II (1891), p. 184-94, p. 189 propose une date de 1548-49, tout comme Anna Bedon, Il Campidoglio : storia di un monumento civile nella Roma papale, Milan, Electa, 2008, p. 117, note 79 (avec ample bibliographie précédente).

2 Ackerman, op. cit., p. 55.

3 Timothy A. Riggs, Hieronymus Cock, Printmaker and Publisher, Londres et New York, Garland, 1977, p. 30 et 238. Les quelques dessins de ruines romaines attribués à Cock [cat. D-2 à D-4] sont des compositions achevées qui ne furent pas réalisées d'après nature, mais à partir d'autres dessins, peut-être de la main de Cock ou d'autres artistes flamands, très certainement Maarten van Heemskerck.

4 Ibid., p. 41, note 23.

5 «La veduta parmi non possa mostrare un certo momento della ricostruzione, ma sembra essere prettamente ideale ». Adolf Michaelis, Storia della collezione capitolina di antichità fino all'inaugurazione del museo (1734) (Mittheilungen des Kaiserlich Deutschen Archaeologischen Instituts, Roemische Abtheilung, vol. VI), Rome, Loescher, 1891, p. 28, note 80 . Cette opinion est partagée dans des études plus récentes qui suggèrent que la gravure a été réalisée à partir d'un assemblage de dessins exécutés à des moments différents. Voir Maria Mura Sommella, "Il monumento di Marco Aurelio in Campidoglio e la trasformazione del Palazzo Senatorio alla metà del Cinquecento ", dans Marco Aurelio. Storia di un monumento e del suo restauro, Cinisello Balsano (Milan), Silvana editoriale, 1989, p. 185 et note 44, analyse reprise par Bedon, op. cit., p. 117 , note 79.

6 OPERVM ANTIQVORVM ROMANORVM / HINC INDE PER DIVERSAS EVROPAE / REGIONES EXTRVCTORVM RELI-/ QVIAS AC RVINIS SAECVLIS OMNI-IBVS SVSPICIENDAS NON MINVS VERE, QVAM PVLCHERRIME DEFORMATAS / LIBELLVS HIC NOVVS CONTINET / Hieronymus Cock piktor anuerpianus / excudebat / CVM GRATIA ET PRIVILEGIO RE./ 1562.

7 « Die Veduten bei Hier. Kock ... zeigen, wie hoch die Verschüttung der Ruinen ging, lehren aber sonst wenig ". Christian Hülsen, Topographie der Stadt Rom im Alterthum, Berlin, Weidmannsche Buchhandlung, 1907, 2 vol., t. I, p. 30, note 3. Une majorité d'historiens qui commentent les gravures de Cock insistent sur l'adéquation ou non des vues à la " réalité " topographique. Voir par exemple Elise Zadek, Der Palatin in den Publikationen Hieronymus Cocks, Magisterarbeit, Humboldt Universität zu Berlin, 2005 [http://edoc.hu-berlin.de/master/zadek-elise-2005-05-13/ HTML/front.html] (avec ample bibliographie précédente).

8 Boudewijn Bakker, "Pictores, Adeste! Hieronymus Cock Recommending His Print Series ", Simiolus: Netherlands Quarterly for the History of Art, vol. 33, no 1/2, Nine Offerings for Jan Piet Filedt Kok, 2007/2008, p. 53-66, p. 60-2 et 64. Sur l'importance du concept de "vérité " (vero en italien) dans les vues topographiques à la Renaissance, voir notamment Lucia Nuti, «The Perspective Plan in the Sixteenth Century. The Invention of a Representational Language ", The Art Bulletin, vol. 76, 1994, p. 105-28, p. 107-109.

9 Sur ce tableau et son contexte, voir notamment Nicole Dacos, Roma quanta fuit. Tre pittori fiamminghi nella Domus Aurea, Rome, Donzelli, 1995, 2 éd. revue et augmentée, Rome, Donzelli, 2001 ; éd. française, Roma quanta fuit ou l'invention du paysage de ruines, Bruxelles et Paris, Musée de la Maison d'Érasme et Somogy Éditions d'art, 2004, ainsi que Michael P. Kemling, " Portrait of the Artist as Michelangelo : Maarten van Heemskerck's Self-Portrait with the Colosseum ", Athanor, vol. 24, 2006, p. 15-21 et Arthur J. DiFuria, "Remembering the Eternal in 1553 : Maerten van Heemskerck's Self-Portrait before the Colosseum ", dans H. Perry Chapman et Joanna Woodall, éd., Envisioning the Artist in the Early Modern Netherlands, Zwolle, Waanders, 2010 (Nederlands kunsthistorisch jaarboek, 59, 2009), p. 90-109.

10 Denis Ribouillault, "Toward an Archaeology of the Gaze : The Perception and Function of Garden Views in Italian Renaissance Villas ", dans Mirka Beneš et Michael G. Lee, éd., Clio in the Italian Garden. Twenty-First-Century Studies in Historical Methods and Theoretical Perspectives, Dumbarton Oaks Colloquium on the History of Landscape Architecture XXXII, Washington, DC, Harvard University Press, 2011, p. 203-32. La distinction entre " document » et «monument " établie par Erwin Panofsky et discutée par Michel Foucault est mentionnée p. 203-205, 226.

11 Riggs, op. cit., p. 156.

12 Karel van Mander, Le livre des peintres de Carel van Mander. Vie des Peintres flamands, hollandais et allemands [1604], traduction, notes et commentaires par Henri Hymans, Paris, J. Rouam, 1884, 2 vol., t. I, p. 306.

13 Sur Saint-Pierre comme ruine dans les dessins de Heemskerck, voir Christoph Thoenes, «St Peter als Ruine : Zu einigen Veduten Heemskercks ", Zeitschrift für Kunstgeschichte, vol. 49, no 4, 1986, p. 481-501. La gravure figurant Saint-Pierre en construction dans la série de Cock de 1561 porte le titre «Aedis D. Petri Romane deformatio". Voir Riggs, op. cit., p. 156 et cat. 109, p. 298 ; Thoenes, op. cit., p. 496-97.

14 Jakob Burckhardt, The Civilization of the Renaissance in Italy, New York, Harper Torchbook edition, 1958, t. I, p. 183-88. Parmi une littérature très fournie sur le sujet, on signalera la belle synthèse de Sabine Forrero-Mendoza, Le temps des ruines. Le goût des ruines et les formes de la conscience historique à la Renaissance, Paris, Champ Vallon, 2002, en particulier p. 112 et ss.

15 Riggs, op. cit., cat. II, 1a, p. 256-57 : «Barbaricus furor, annorumque horrenda vorago, / Sic Orbis Reginam, illam, lachrimabile, Romam / Vastarunt, fatis nimirum vrgentibus : ecquae / Seruandis reliquis vsquam fiducia regnis?".

16 Christopher P. Heuer, "Hieronymus Cock's Aesthetic of Collapse », Oxford Art Journal, vol. 32, no 3, 2009, p. 387-408. 
17 Dans le catalogue de Riggs ainsi que ceux des musées qui possèdent des gravures de Cock, les titres des gravures sont systématiquement liés aux ruines et aux monuments, même si la plupart d'entre eux ne sont pas clairement identifiés ni identifiables. À part les dessinateurs, les personnages ne sont jamais mentionnés. Je n'ai repéré que deux études qui abordent succintement la question des personnages dans les séries des ruines de Cock. Voir Gisela Barche, "Studien zur Darstellung der Stadt Rom (1750-1870) ", thèse de doctorat, Munich, 1985, p. 183-84 et Stephanie Gropp, «Das Kolosseum in der Druckgraphik des 15. bis 19. Jahrhunderts ", thèse de doctorat, Bonn, 2012, p. 175-77. Sur les modalités du regard porté sur le paysage, voir les remarques de Daniel Arasse, Le détail. Pour une histoire rapprochée de la peinture [1992], Paris, Champs Flammarion, 1996, p. 248-52.

18 Sur cette vue et les décors du palais des Conservateurs, voir Denis Ribouillault, "Landscape all'antica and Topographical Anachronism in Roman Fresco Painting of the Sixteenth Century ", Journal of the Warburg and Courtauld Institutes, vol. 71, 2008, p. 211-37, p. 216-19.

19 Sur les statues des dieux-fleuves du Capitole, voir Michaelis, Storia della collezione, op. cit., p. 25-6, fig. 1 ; Rodolfo Lanciani, Storia degli scavi di Roma e notizie intorno le collezione romane di antichità, $2^{e}$ éd., Rome, Loescher, 1989-94, t. I, p. 241 ; Phyllis P. Bober et Ruth Rubinstein, Renaissance Artists and Antique Sculpture, Londres, Harvey Miller, 1986, p. 99-104, en particulier 101-102 (n. 65) ; Serena Ensoli Vittozzi, «La decorazione scultorea della Scalinata del Palazzo Senatorio ", dans Maria Elisa Tittoni, éd., La facciata del Palazzo Senatorio in Campidoglio, Pisa, Ospedaletto, 1994, p. 101-34, p. 104-18 (avec ample bibliographie et documentation) ; Claudia Lazzaro, "River gods : Personifying Nature in Sixteenth-Century Italy ", Renaissance Studies, vol. 25, no 1, 2011, p. 70-94, p. 78. Sur les dieux-fleuves à la Renaissance, voir aussi Ruth Rubinstein, "The Renaissance Discovery of Antique River-God Personifications ", dans Cristina De Benedictis, éd., Scritti di storia dell'arte in onore di Roberto Salvini, Florence, Sansoni, 1984, p. 257-63.

20 «Ma hoggi amendue questi simulacri [les dieux-fleuves] si tolgono da questo luogo, per riporli davanti al palagio del Campidoglio, e ne hanno già tolto via uno ". Lucio Fauno, Antichità della città di Roma, Rome, 1553, f. 38b ; Michaelis, Storia della collezione, op. cit., p. 29-30.

21 Hans H. Brummer, The Statue Court in the Vatican Belvedere, Stockholm, Almqvist \& Wiksell, 1970, p. 191-92 ; Lazzaro, op. cit., p. 71-3.

22 Ruth Rubinstein, " "Tempus edax rerum” : A Newly Discovered Painting by Hermannus Posthumus ", The Burlington Magazine, vol. 127 , no 988 , juillet 1985 , p. $425-33$ et $435-36$, p. 430 , cat. 50-2.

23 Ruth Rubinstein, "The Statue of the River God Tigris or Arno ", dans Matthias Winner, Bernard Andreae et Carlo Pietrangeli, éd.,
Il Cortile delle Statue : Der Statuenhof des Belvedere im Vatikan, Mayence, P. von Zabern, 1998, p. 275-85.

24 Lazzaro, op. cit. Sur les interprétations variées données aux statues antiques à la Renaissance voir, par exemple, Leonard Barkan, "The Beholder's Tale : Ancient Sculpture, Renaissance Narratives ", Representations, vol. 44, 1993, p. 133-66.

25 Una Roman d'Elia, "Giambologna's Giant and the Cinquecento Villa Garden as a Landscape of Suffering ", Studies in the History of Gardens \& Designed Landscapes, vol. 31, no 1, 2011, p. 1-25, p. 7-16 ; Rubinstein, "The Statue of the River God Tigris or Arno ", op. cit., p. 281-83.

26 Lazzaro, op. cit., p. 80-3 ; Hubert Damish, Le Jugement de Pâris, Paris, Flammarion, 1992, p. 53-76.

27 Ulisse Aldrovandi, "Delle statue antiche che per tutta Roma in diversi luoghi, e case si veggono ", dans Lucio Mauro, Le antichità della città di Roma brevissima raccolte da chiunque ne ha scritto, $3^{\mathrm{e}}$ éd., Venise, 1562, p. 115-315, p. 270-72 ; Michaelis, Storia della collezione, op. cit., p. 30 ; Serena Ensoli Vittozzi, Musei Capitolini. La collezione egizia, Cinisello Balsamo (Milan), Silvana editoriale, 1990, p. 12-13, note 5, fig. 4-5 ; Brian A. Curran, "The Sphinx in the City : Egyptian Memories and Urban Spaces in Renaissance Rome (and Viterbo)", dans Stephen J. Campbell et Stephen J. Milner, éd., Artistic Exchange and Cultural Translation in the Italian Renaissance City, Cambridge, Cambridge University Press, 2004, p. 294-326, p. 307-08 (avec bibliographie sur les sphinx à la note 98 ).

28 Fritz Saxl, "The Capitol during the Renaissance : A Symbol of the Imperial Idea ", repris dans Lectures, 2 vol., Londres, The Warburg Institute, 1957, t. I, p. 200-14 ; Angela Marino, «Idoli e colossi : la statuaria antica sulla piazza del Campidoglio da Sisto IV a Leone X ", dans Silvia Danesi Squarzina, éd., Roma, centro ideale della cultura dell'Antico nei secoli XV e XVI : da Martino $V$ al sacco di Roma 1417-1527, Milan, Electa, 1989, p. 237-47. Sur l'intérêt de Jules Romain pour les sphinx du Capitole, voir Bertrand Jaeger, "La Loggia delle Muse nel Palazzo Te e la reviviscenza dell'Egitto Antico nel Rinascimento ", dans Mantova e l'Antico Egitto da Giulio Romano a Giuseppe Acerbi, Florence, Olschki, 1994, p. 21-39.

29 Sur les visites de collections d'Antiquité à Rome à la Renaissance, voir William Stenhouse, "Visitors, Display, and Reception in the Antiquity Collections of Late-Renaissance Rome ", Renaissance Quarterly, vol. 58, no 2, 2005, p. 397-434. Sur le Paragone, voir Sefy Hendler, La guerre des arts. Le paragone, sa pratique peinturesculpture, en Italie, $X V^{\mathrm{e}}-X V I I^{\mathrm{e}}$ siècle, Rome, "L'Erma di Bretschneider ", 2012.

30 Sur les associations symboliques, politiques et religieuses liées aux monuments équestres de l'Antiquité à la Renaissance, voir, parmi une riche bibliographie, H.W. Janson, "The Equestrian Monument from Cangrande della Scala to Peter the Great ", repris dans Sixteen Studies, New York, Abrams, 1973, p. 157-88.

31 Lettre de Giovanni Maria della Porta, ambassadeur du duc d'Urbin 
à Rome, datée de 1538 : «Il Papa fa levare il cavallo di Sto Janno laterano et porlo sulla piazza di Campidoglio mostrando che sia motivo dei Romani... Michelangelo contrastò assai, per quanto lui mi dice, che questo cavallo non se levasse, parendogli che'l stesse meglio dove l'era et che, se lui non havesse tanto disuasi il papa, che S. stà voleva similmente levare gli dui cavalli e statue di Montecavallo », citée dans Georg Gronau, «Die Kunstbestrebungen der Herzöge von Urbino : II-III ", Jahrbuch der Preussischen Kunstsammlungen, vol. 27, 1906, 1-11, 12-44, p. 9, n. XXI s.d..

32 Sur les destructions des antiquités de Rome au XVI ${ }^{\mathrm{e}}$ siècle, voir notamment Ribouillault, "Landscape all'antica », op. cit., p. 216-18; Heuer, op. cit., p. 396 et ss.

33 Voir, parmi une vaste bibliographie, Margaret M. McGowan, The Vision of Rome in Late Renaissance France, New Haven et Londres, Yale University Press, 2000, p. 187-227 (sur du Bellay) et chap. 7, "Negative Responses and Reverse Appropriation", p. 251-82.

34 Le motif du kakker apparaît dans chaque série, au moins une fois : voir, par exemple, Riggs 3, 15 (1551) et Riggs 130 (1562).

35 Jeff Persels, " "The Mass and the Fart Are Sisters" : Scatology and Calvinist Rhetoric against the Mass, 1500-1563 » et Josef Schmidt et Mary Simon, "Holy and Unholy Shit : The Pragmatic Context of Scatological Curses in Early Modern German Reformation Satire ", dans Jeff Persels et Russell Ganim, éd., Fecal Matters in Early Modern Literature and Art : Studies in Scatology, Aldershot, Ashgate, 2004, p. 38-55 et p. 109-17.

36 Van Mander, Le livre des peintres, op. cit., t. I, p. 192. Sur le kakker chez Patinir, voir Michel Weemans, "Les rhyparographes ", dans Rires et autres éclats (Parade. Revue d'art et de littérature, no 8), Tourcoing, École régionale supérieure d'expression plastique, 2008, p. 72-91, p. 77 et Reindert Falkenburg, « Le diable est dans le détail. Manières de voir les paysages cosmiques de Joachim Patinir ", dans Denis Ribouillault et Michel Weemans, éd., Le paysage sacré. Le paysage comme exégèse dans l'Europe de la première modernité, Giardini e paesaggio 29, Florence, Olschki, 2011, p. 3-25, p. 3.

37 Sur les rapports entre Cock et Bruegel, voir Riggs, op. cit., p. 50-2, 135, 183-85 ; Timothy A. Riggs, "Bruegel and His Publisher ", dans Otto G. von Simson et Matthias Winner, éd., Pieter Bruegel und seine Welt, Berlin, Mann, 1979, p. 165-73 ; Nadine M. Orenstein, «Images to Print : Pieter Bruegel's Engagement with Printmaking ", dans Nadine M. Orenstein, éd., Pieter Bruegel the Elder. Drawings and Prints, catalogue d'exposition, New York, Metropolitan Museum of Art, New Haven et Londres, Yale University Press, 2001, p. 41-55 ; Weemans, op. cit., p. 78-84. Sur le rhyparographe dans l'art flamand du premier âge moderne, voir aussi Norman Bryson, Looking at the Overlooked, Londres, Reaktion Books, 1990, p. 60-95 ; Reindert Falkenburg, "Pieter Aertsen, Rhyparographer ", dans Jelle Koopmans et Mark Meadow, éd., Rhetoric - rhétoriqueurs - rederijkers, Amsterdam, Royal Netherlandish Academy of Arts and Sciences, 1995, p. 197-215 ; Marga- ret A. Sullivan, «Aertsen's Kitchen and Market Scenes : Audience and Innovation in Northern Art ", The Art Bulletin, vol. 81, no 2, juin 1999, p. 236-66.

38 Weemans, op. cit., p. 84, fig. 7, signale la présence du kakker. Sur la tour de Babel comme travestissement de l'image du Colisée et les diverses interprétations du thème dans l'œuvre de Bruegel, voir Walter S. Gibson, Mirror of the Earth. The World Landscape in Sixteenth-Century Flemish Painting, Princeton, Princeton University Press, 1989, chap. 5.

39 Weemans, op. cit., p. 77.

40 Lucas de Heere, Invective, an eenen Quidam schilder : de welcke beschimpte de Schilders van Handwerpen, dans Den Hof en Boomgaerd der Poesien (Ghent, 1565), éd. W. Waterschoot, Zwolle, W.E.J. Tjeenk Willink, 1969, vol. 66, p. 87-9 (édition électronique (2002) disponible sur http://www.dbnl. org/tekst/_jaa005196401_01/_jaa005196401_01_0002.php. Le poème est signalé dans Weemans, op. cit., p. 80 et discuté notamment dans David Freedberg, "Allusion and Topicality in the Work of Pieter Bruegel : The Implications of a Forgotten Polemic ", dans The Prints of Pieter Bruegel the Elder, David Freedberg, éd., catalogue d'exposition (Tokyo Shimbun), Tokyo, 1989, p. 53-65 et Mark A. Meadow, "Bruegel's Procession to Calvary, Æmulatio and the Space of Vernacular Style ", dans Jan de Jong et Mark Meadow, éd., Pieter Bruegel, Zwolle, Waanders, 1997 (Nederlands Kunsthistorisch Jaarboek, vol. 47, 1997), p. 181-205, avec une traduction en anglais p. 181-82. Cock publia deux séries sur les Arts libéraux d'après Frans Floris. Il grava lui-même la première en 1550 (Riggs, op. cit., cat. II. A. 26-35). La seconde, gravée en 1565 par Cornelis Cort d'après des dessins de Symon Jansz. Kies (ibid., p. 330), reprenait le cycle de tableaux réalisés pour la villa de campagne du grand collectionneur anversois Nicolas Jonghelinck, lequel avait aussi commandé à Pieter Bruegel la fameuse série des Saisons. C’est dans le contexte de cette mise en compétition des deux peintres rivaux que s'insère le poème de Lucas de Heere.

41 Riggs, op. cit., p. 50-1. Pour une analyse de la peinture de paysage de Bruegel comme une réponse à l'art et à la théorie de l'art italiens, voir notamment Jane ten Brink Goldsmith, « Pieter Bruegel the Elder and the Matter of Italy ", Sixteenth Century Journal, vol. 23, no 2, 1992, p. 205-34, p. 206 : « Notably, Bruegel's association with Cock began with what may be understood as a revival of Netherlandish art of the early sixteenth century ".

42 Van Mander, Le livre des peintres, op. cit., t. I, p. 299.

43 "The epithet "Peasant Bruegel" still clings tenaciously to Pieter Bruegel the Elder », écrit Martin Royalton-Kisch, «Pieter Bruegel as a Draftsman : The Changing Image ", dans Orenstein, éd., Pieter Bruegel the Elder. Drawings and Prints, op. cit., p. 13-39, p. 13 et p. 36, n.1.

44 Weemans, op. cit., p. 77 pour la citation. Sur la dimension " pittoresque " plutôt qu' " archéologique " des ruines de Cock, voir les 
remarques de Riggs, op. cit., p. 167-68 : "Cock's prints are the first to present views of genuine Roman ruins in the context of picturesque landscape... ". Sur l'importance du paysage dans les gravures publiées par Cock à partir de 1555, voir ibid., p. 183-86.

45 Van Mander, Le livre des peintres, op. cit., t. I, p. 103. Pour une analyse approfondie de ce passage avec la bibliographie correspondante, voir Todd M. Richardson, Pieter Bruegel the Elder. Art Discourse in the Sixteenth-Century Netherlands, Farnham, Ashgate, 2011, p. 205-14.

46 «En effet, il est peu d'œuvres de sa main qu'on puisse regarder sans rire, et le spectateur le plus morose se déride à leur aspect ». Van Mander, Le livre des peintres, op. cit., t. I, p. 299.

47 Ibid., t. I, p. 290 ; Riggs, op. cit., p. 31, 210-11; Walter S. Gibson, "Artists and Rederijkers in the Age of Bruegel ", The Art Bulletin, vol. 63, no 3, septembre 1981, p. 426-46, p. 431. Sur Bruegel et les rederijkers, voir notamment Bart A.M. Ramakers, "Bruegel en de rederijkers : Schilderkunst en literatuur in de zestiende eeuw ", dans Jan de Jong et Mark Meadow, éd., Pieter Bruegel, Zwolle, Waanders, 1997 (Nederlands Kunsthistorisch Jaarboek, vol. 47, 1997), p. 80-105. Sur la dimension comique dans l'œuvre de Bruegel, voir la contribution récente de Walter S. Gibson, Pieter Bruegel and the Art of Laughter, Berkeley, University of California Press, 2006 (avec ample bibliographie précédente).

48 Sur ce point, voir notamment Jürgen Müller, « Albrecht Dürer's Peasant Engravings : A Different Laocoön, or the Birth of Aesthetic Subversion in the Spirit of the Reformation ", Journal of Historians of Netherlandish Art, vol. 3, no 1, 2011 [www.jnha.org]. Il reste cependant difficile de déterminer si les gravures de Cock comportent ce type de commentaire religieux, même si on ne peut l'exclure.

49 Il existe de nombreuses « citations " (généralement subversives) de l'art italien et antique dans l'œuvre de Bruegel. Sur le sujet, voir notamment Goldsmith, op. cit. Sur Aertsen, voir la contribution centrale de Falkenburg, Pieter Aertsen, op. cit. reprise et développée par Sullivan, op. cit. Sur la faible opinion italienne de l'art flamand à la Renaissance, voir notamment Sylvie Deswartes Rosa, "Si dipinge col cervello e non con le mani ", dans Fiamminghi a Roma 1508-1608, actes du colloque (Bruxelles, 1995), Nicole Dacos, éd. (Bollettino d'arte, supplemento al no 100), 1997, p. 277-94.

50 Müller, op. cit.

51 Voir Giuliano Briganti, Ludovica Trezzani, Laura Laureati, I Bamboccianti : Pittori della vita quotidiana a Roma nel Seicento, Roma, Bozzi, 1983 et surtout, pour son analyse de la subversion flamande de la tradition antique et italienne chez Pieter van Laer, David A. Levine, "The Roman Limekilns of the Bamboccianti ", The Art Bulletin, vol. 70, no 4, décembre 1988, p. 569-89. Pour une discussion éclairante de la critique satirique de Rome et de sa culture dans l'art et la littérature du XVII e siècle, voir Frédéric Cousinié, "Rome ridicule. Inversion, souillure et dégradation du modèle romain ", dans Marc Bayard, éd., Rome - Paris, 1640. Transferts culturels et renaissance d'un centre artistique, Paris, Somogy, 2010, p. 77-105. Du même auteur, on peut consulter Esthétique des fluides. Sang, sperme, merde dans la peinture française du XVII siècle, Paris, Félin, 2011.

52 Cousinié, Rome ridicule, op. cit., p. 83-90, «Le Lorrain Bamboche». Sur ce même sujet, voir Sheila McTighe, The Imaginary Everyday : Genre Painting and Prints in Italy and France, 1580-1670, New York, The Brownes Library, 2007, chap. 5. Dans sa Teutsche Akademie (1675), Joachim von Sandrart rapporte que Claude se rendait pour dessiner dans la campagne romaine en compagnie d'autres artistes, notamment Pieter van Laer. Sur la relation de Claude à Van Laer, voir notamment A. Janeck, « Naturalismus und Realismus : Untersuchungen zur Darstellung der Natur bei Pieter van Laer und Claude Lorrain ", Storia dell'arte, vol. 26 / 28, 1976, p. 285-304.

53 Sur le kakker dans les tableaux de Claude, voir Cousinié, Rome ridicule, op. cit., p. 85-9. Sur le dessinateur, voir Denis Ribouillault, "De la pratique au mythe : la figure du dessinateur dans les paysages de Claude Lorrain ", dans Anne-Laure Imbert, éd., Flâneurs et regardeurs dans la peinture de la Renaissance, Actes du colloque (Paris, I.N.H.A., juin 2009), à paraître.

54 Voir, sur ce tableau, l'interprétation convaincante de McTighe, $o p$. cit., p. 321 et sq. Lallusion à Michel-Ange fait référence au fameux Dialogues de Francesco da Holanda, dans lequel le célèbre artiste critique la peinture flamande et en particulier la peinture de paysage et conclut «Les œuvres que l'on fait en Italie sont presque les seules que nous puissions appeler de vraies peintures, c'est pour cela que nous appelons italienne la bonne peinture... ». François de Hollande, De la peinture : dialogues avec Michel-Ange, traduit de l'italien par Simone Matarasso-Gervais, Aix-en-Provence, Alinea, 1984, p. 31-3. Sur ce sujet, voir Deswartes-Rosa, op. cit. 